\title{
A lipid droplet-peroxisome network drives longevity by monounsaturated fatty acids via modulating ether lipid synthesis and ferroptosis
}

Anne Brunet ( $\square$ anne.brunet@stanford.edu )

Stanford University https://orcid.org/0000-0002-4608-6845

Katharina Papsdorf

Stanford University

Amir Hosseini

Stanford University

Jason Miklas

Stanford University https://orcid.org/0000-0002-9002-0667

Matias Cabruja

Stanford University

Yong Yu

Xiamen University

Luke Meraz Murphy

Stanford University

Carlos Silva-Garcia

Harvard T. H. Chan School of Public Health

Pallas Yao

Harvard University

Elisa de Launoit

Stanford University

William Mair

Harvard University https://orcid.org/0000-0002-0661-1342

Meng Wang

Baylor College of Medicine/Howard Hughes Medical Institute https://orcid.org/0000-0002-5898-6007

Michael Snyder

Stanford University https://orcid.org/0000-0003-0784-7987

\section{Biological Sciences - Article}

Keywords: mono-unsaturated fatty acids, organelle hub, lipid droplet 
Posted Date: August 13th, 2021

DOl: https://doi.org/10.21203/rs.3.rs-740521/v1

License: (c) (i) This work is licensed under a Creative Commons Attribution 4.0 International License. Read Full License 

fatty acids via modulating ether lipid synthesis and ferroptosis

3 Katharina Papsdorf ${ }^{1}$, Amir Hosseini ${ }^{1}$, Jason W. Miklas ${ }^{1}$, Matias Cabruja ${ }^{1}$, Yong Yu ${ }^{2,3}$, Luke

Meraz Murphy ${ }^{1}$, Carlos Silva Garcia ${ }^{4}$, Pallas $\mathrm{Yao}^{4}$, Elisa de Launoit ${ }^{1}$, William B. Mair ${ }^{4}$, Meng

C. Wang ${ }^{2,5}$, Michael P. Snyder ${ }^{1}$, Anne Brunet ${ }^{1,6, \#}$

${ }^{1}$ Department of Genetics, Stanford University, Stanford, CA94305, USA

${ }^{2}$ Department of Molecular and Human Genetics, Huffington Center on Aging, Baylor College of Medicine, Houston, TX77030, USA

${ }^{3}$ Present address: School of Life Sciences, Xiamen University, China

${ }^{4}$ Department of Molecular Metabolism, Harvard, Boston, MA02115, USA

${ }^{5}$ Howard Hughes Medical Institute, Baylor College of Medicine, Houston, TX77030, USA

${ }^{6}$ Glenn Laboratories for the Biology of Aging, Stanford University, Stanford, CA94305, USA

\#Correspondence: Anne Brunet, anne.brunet@stanford.edu

14 Abstract

15 Dietary mono-unsaturated fatty acids (MUFAs) are linked to human longevity and extend lifespan in several species ${ }^{1-12}$. But the mechanisms by which MUFAs promote longevity remain unclear. Here we show that an organelle hub involving lipid droplets and peroxisomes is critical for lifespan extension by MUFAs in C. elegans. MUFA accumulation increases lipid droplet number in fat storage tissues, and lipid droplet synthesis is necessary for MUFA-mediated longevity. Interestingly, the number of lipid droplets in young individuals can predict their remaining lifespan. MUFA accumulation also increases the number of peroxisomes, and peroxisome activity is required for MUFA-mediated longevity. By performing a targeted screen, we uncover a functional network between lipid droplets and peroxisomes in longevity. Interestingly, our screen also identifies ether lipids as critical components of the lipid dropletperoxisome network. Using lipidomics, we find that the ratio of MUFAs to polyunsaturated fatty acids (PUFAs) in ether lipids is increased by MUFA accumulation. Ether lipids are involved in ferroptosis, a non-apoptotic form of cell death ${ }^{13-17}$, and MUFAs promote longevity in part via suppression of ferroptosis. Our results identify a mechanism of action for MUFAs to extend lifespan and uncover an organelle network involved in the homeostasis of MUFA-rich ether lipids. Our work also opens new avenues for lipid-based interventions to delay aging. 
Lifespan is strongly influenced by diet. High fat diets are mostly detrimental for lifespan, but specific lipids can be beneficial for health and longevity ${ }^{18-21}$. Diets that are rich in monounsaturated fatty acids (MUFAs), such as olive oil in the Mediterranean diet, are correlated with longevity in humans ${ }^{1,2}$. Specific MUFAs (e.g. oleic acid and palmitoleic acid) causally extend lifespan in species ranging from $C$. elegans to mammals ${ }^{3,4,6-11}$. Yet the mechanism by which some lipids promote longevity, while others are detrimental for health, remain unknown.

Attractive candidates for the mechanism of MUFA action are conserved organelles involved in fat storage and metabolism, such as lipid droplets ${ }^{22-24}$. While the role of lipid droplets has started to be evaluated during aging, age-related diseases, and the response to stressors in different species ${ }^{25-34}$, it is still unclear whether these organelles are positive or negative regulators of health. However, the importance of lipid droplets in MUFA-mediated longevity, and their mechanism of action for lifespan extension, is unknown.

We asked whether MUFAs influence lipid droplets in C. elegans, and whether this is critical for lifespan extension. To precisely assess lipid droplet number, we used Stimulated Raman Scattering (SRS) - a spectroscopy method that enables label-free imaging of lipids by visualizing carbo-hydrogen bonds ${ }^{35-39}$. MUFA accumulation was induced by upregulating SCD1/FAT-7, the enzyme that produces MUFAs (via knock-down of the chromatin regulator ash-2 [ref $\left.\mathrm{r}^{6}\right]$ ), or by downregulating FAT-2, the enzyme that catabolizes MUFAs ${ }^{6,10}$ (Fig. 1a). These manipulations indeed led to lifespan extension and MUFA accumulation (Extended Data Fig.1a, b, c) $)^{5,6,10}$. Interestingly, MUFA accumulation by ash-2 or fat-2 knock-down resulted in increased number of lipid droplets in intestinal cells, the main fat storage cells in C. elegans (Fig. $1 b, c)$. These lipid droplets were not only more numerous, but they also had higher SRS intensity (Extended Data Fig. 1d), indicating they were more packed with lipids. We independently confirmed that MUFA enrichment led to increased lipid droplet number using a transgenic strain that expresses the lipid droplet membrane protein DHS-3 fused to GFP ${ }^{40-42}$ (Fig. 1d, e). Males also showed increased lipid droplet number in the intestine upon MUFA accumulation (Fig. 1f, Extended Data Fig. 1e), indicating the effect of MUFAs on lipid droplets generalizes across sexes. MUFA accumulation led to lipid droplet accumulation in intestine cells, but not in other cells (e.g. hypodermis or eggs) (Fig. 1g, h, Extended Data Fig. 1f, g), and it did not impact lipid 
droplet size in a uniform manner (Extended Data Fig. 1h, i). Thus, MUFA accumulation results in increased lipid droplet number in lipid storage tissues.

Importantly, dietary supplementation with oleic acid, a cis MUFA present in olive oil and nuts, increased intestinal lipid droplet number and extended lifespan (Fig. 1i, j, k, 1). In contrast, dietary supplementation with elaidic acid, a trans MUFA present in margarine and dairy and known to have detrimental effects on human health ${ }^{43}$, did not increase lipid droplet number and did not extend lifespan (Fig. 1j, k, 1). Hence, cis MUFA (but not trans MUFA) supplementation triggers an increase lipid droplet number, which correlates with lifespan extension.

To test if increased lipid droplet number is necessary for MUFAs to extend lifespan, we inhibited lipid droplet synthesis by targeting genes involved in this process (Fig. 1m). Knockdown of LIPIN1/lpin-1, which is important for the synthesis of lipid droplets ${ }^{44-50}$ (Fig. 1n), resulted in fewer lipid droplets in both basal and MUFA-enriched conditions (Fig. 1n, o, Extended Fig. 1j, 1). Interestingly, lpin-1 deficiency blunted longevity by MUFA accumulation due to ash-2 depletion (Fig. 1p) or oleic acid supplementation (Fig. 1q). Consistently, deficiency in SEIPIN/seip-1, which is implicated in the early steps of lipid droplet biogenesis ${ }^{51-55}$ (Fig. 1m), also abolished lipid droplet increase in response to MUFA accumulation by ash-2 RNAi (Extended Fig. 1m, n), and seip-1 mutants no longer exhibit lifespan extension in response to MUFA accumulation by ash-2 or fat-2 RNAi (Fig. 1r, s). Collectively, our data indicate that an increase in lipid droplet number is necessary for MUFAs to extend lifespan. remaining lifespan in a population of individuals? Depletion of HSL1/hosl-1 or ATGL/atgl-1, which are involved in lipid droplet hydrolysis (see Fig. $1 \mathrm{~m})^{24,56-58}$, led to an increase in lipid droplet number and a slight but significant lifespan extension (Fig. 2a, b, Extended Data Fig. 2a, b). These data suggest that increased lipid droplet number is sufficient to extend lifespan, even in the absence of MUFAs.

To determine if a high lipid droplet number can predict a long lifespan, we assessed the lifespan of genetically identical individuals with varying amounts of lipid droplets in a population of $C$. elegans individuals. We used the large particle BioSorter ${ }^{59,60}$ to sort two subpopulations of young adult worms expressing high or low fluorescent levels of the lipid droplet marker fused to GFP (Fig. 2c, Extended Data Fig. 2c). Higher fluorescence worms 
indeed had more lipid droplets compared to lower fluorescence ones (Fig. 2d, e). Interestingly, individuals with more lipid droplets lived significantly longer than individuals with less lipid droplets (Fig. 2f). The predictive power of lipid droplet number for longevity was even more evident when the worms were sorted at middle age (Fig. 2g, h, Extended Data Fig. 2d). Thus, increased lipid droplet number is sufficient to extend lifespan and can predict remaining lifespan, consistent with a beneficial role for lipid droplets in longevity.

We next probed the mechanism by which lipid droplets extend lifespan in response to MUFAs. Lipid droplets interact with other organelles, such as mitochondria and peroxisomes, to regulate lipid metabolism ${ }^{23,61-65}$ (Fig. 3a). Re-analysis of transcriptomic datasets of C. elegans with or without MUFA accumulation ${ }^{5,6}$ showed peroxisome-related GO terms in conditions of MUFA accumulation (Fig. 3b). Peroxisomes have been shown to regulate longevity in $C$. elegans and Drosophila in some contexts ${ }^{66,67}$, but their role in longevity by dietary fatty acids and their relationship with lipid droplets for lifespan extension is unknown. Using transgenic reporter strain that carries GFP fused to a peroxisome import signal ${ }^{66,68}$, we found that MUFA accumulation due to ash-2 and fat-2 depletion led to increased number and intensity of peroxisomes (Fig. 3c, d, Extended Data Fig. 3a, b, c, d). Depletion of the peroxisomal protein importer prx-5 or the peroxisome membrane protein importer prx-19 blunted peroxisome increase in response to MUFAs (Extended Data Fig. 3e), and this deficiency in peroxisome activity abolished longevity due to ash-2 knock-down or dietary oleic acid supplementation (Fig. 3e, Extended Data Fig. 3f, g). Hence, peroxisome activity is required for longevity in response to MUFA accumulation.

We next examined the relationship between lipid droplets and peroxisomes in response to MUFA accumulation. Lipid droplet number correlated with peroxisome number in individual worms (Extended Data Fig. 3h). Furthermore, both organelles exhibited similar dynamics with age and in response to MUFA accumulation (Fig. 3f), with an increase in young adults followed by a decrease at middle age (Extended Data Fig. 3i, j).

Lipid droplets and peroxisomes can form physical contacts ${ }^{65,69}$, but how they influence each other functionally - especially in the context of longevity - remains unknown. To understand the functional connection between lipid droplets and peroxisomes, we performed a targeted screen to identify genes that impact lipid droplet and peroxisome number. We tested 50 
genes known to be involved in metabolism, or present in lipid droplets or peroxisomes $^{40,41,47,55,65,68,70-82}$ (Fig. 3g). There was a striking correlation between peroxisome and lipid droplet numbers upon perturbation of proteins involved in metabolism, lipid droplet, and peroxisome function (Fig. 3h), consistent with a joint regulation of these organelles. Perturbations that led to more lipid droplets and peroxisomes were often those that resulted in lifespan extension (Fig. 3h). Interestingly, our screen also uncovered the genes ads-1 and fard-1 as hits that, when knocked-down, reduce both lipid droplet and peroxisome numbers (Fig.3i-1). ADS-1 and FARD-1 are involved in the synthesis of a specific form of lipids, called ether lipids, which have an ether bond instead of the more traditional ester bond (see Fig. 4d) ${ }^{79}$. These data reveal a functional hub between lipid droplets and peroxisomes in longevity and identify ether lipid synthesis as an important regulator of this network.

Is ether lipid synthesis important for longevity by MUFAs? Deficiency in the ether lipid synthesis gene $a d s-1$ and fard -1 blunted the lifespan extension due to MUFA accumulation upon ash-2 and fat-2 depletion (Fig. 3m, Extended Data Fig. 3k, 1). Ether lipid synthesis also abolished the ability of lipid droplet increase due to hosl-1 depletion or sorting to extend lifespan (Fig. 3n, Extended Data Fig. 3m). While the decrease in lipid droplet and peroxisome number by ads-1 and fard-1 knock-down could lead to this reduction in longevity, these data also raise the possibility that ether lipid synthesis plays a role in longevity by MUFA accumulation. In contrast, depletion of enzymes related to peroxisomal beta oxidation $(\text { daf }-22)^{68,72,83-85}$ or hydrogen peroxide degradation $(c t l-3)^{86}$ were not required for lifespan extension by MUFA accumulation (Extended Data Fig. 3n, o). Collectively, these data reveal a connection between lipid droplets and peroxisomes for longevity and highlight ether lipids as being important for lifespan.

Ether lipids are present in several lipid classes, including triglycerides and phospholipids, and they can modulate ferroptosis ${ }^{13-16}$, a form of cell death due to iron accumulation, in both $C$. elegans and mammalian cells ${ }^{87,88}$ (see Fig. 4g). We first asked how ether lipids (and other lipids) change in response to MUFA accumulation. To this end, we performed lipidomics on worms treated with ash-2 RNAi to induce MUFA accumulation, in the presence or absence of peroxisome deficiency (prx-5 RNAi) (Fig 4a). As expected, ash-2 RNAi led to a global increase in MUFAs and triglycerides (Fig. 4b, Extended Data Fig 4a), and this was abolished when 
peroxisome function was inhibited (Fig. 4b, Extended Data Fig 4a). Principal component analysis (PCA) on all lipid species easily separated samples with MUFA enrichment (ash-2 RNAi) and with peroxisome deficiency (prx-5 RNAi) (Fig. 4c). Many lipids were remodeled in response to MUFA accumulation by ash-2 knock-down with or without peroxisome function (see Extended Data Table 6 and Methods), providing a great resource for lipid composition changes under these conditions. Importantly, ether lipids were one of the major lipids driving the separation between MUFA-enriched samples with and without peroxisome function (Fig. 4c, PC1).

MUFAs and PUFAs can be incorporated into ether lipids (Fig. 4d), and PUFA-containing ether lipids are known to be detrimenta $1^{13,14}$. While MUFA accumulation led to a decrease in total ether lipid levels (Extended Data Fig 4b), it actually increased the MUFA to PUFA ratio among ether lipids (Fig. 4e, f, Extended Data Fig 4c). The class of lipids that contains most ether bonds were the membrane lipids phosphatidylethanolamine (PE) (Extended Data Fig 4d), and MUFA accumulation also led to a significant increase in the MUFA to PUFA ratio among ether lipids in PEs (Fig. 4f, Extended Data Fig 4e). Thus, MUFA accumulation may be beneficial by increasing the ratio of MUFAs to PUFAs among ether lipids, thereby off-setting the negative impact of PUFA-containing ether lipids.

Ether lipids are involved in ferroptosis, an iron-dependent form of cell death characterized by accumulation of lipid peroxides ${ }^{13-15,89,90}$ (Fig. 4g). We asked whether modulating ferroptosis could impact MUFA-mediated longevity. To induce ferroptosis, we depleted the glutathione peroxidase GPX4/gpx-1, which normally protects from lipid peroxidation and ferroptosis ${ }^{91}$ (Fig. 4g). Interestingly, gpx-1 knock-down blocked longevity due to ash-2 knock-down (Fig. 4h), suggesting that protection from ferroptosis is required for MUFA-mediated longevity. To block ferroptosis, we used the iron-chelating drug Salinazid ${ }^{88}$. We found that Salinazid extended C. elegans lifespan, as previously shown ${ }^{88}$, but could not further extend the long lifespan of ash-2 or fat-2 deficient worms (Fig 4i, Extended Data Fig. 4f). These results are consistent with the possibility that MUFAs extend lifespan by inhibiting ferroptosis. Finally, deficiency in lipid droplets (lpin-1 RNAi) or ether lipid synthesis (ads-1 RNAi) abolished Salinazid-mediated longevity (Fig. 4 j, k). These results suggest that the beneficial effect of blocking ferroptosis on lifespan also requires intact lipid metabolism, perhaps because an appropriate ratio of MUFAs vs. PUFAs among ether lipids is critical. Together, our results suggest that MUFAs extend lifespan by increasing the number of lipid droplets and 
peroxisomes, which could impact the ratio of MUFAs vs. PUFAs among ether lipids and result in the inhibition of ferroptotic death.

Our study identifies a mechanism of action by which dietary fatty acids extend lifespan and uncovers the importance of a lipid droplet-peroxisome organelle hub in longevity. We find that high lipid droplet and peroxisome number is beneficial for longevity by monounsaturated fatty acids such as oleic acid (present in olive oil) and a high lipid droplet number in young individuals can even predict remaining longevity. The role of lipid droplets in longevity was not clear, with studies indicating beneficial effects but others showing detrimental effects. For example, in Drosophila, expression of a protein that tethers lipid droplets is beneficial for shortterm survival in response to starvation and is associated with high median lifespan ${ }^{30}$, and lipid droplet composition can protect stem cell niches from damaging lipid peroxidation ${ }^{29}$. Moreover, lipid droplets can release MUFAs, which in turn activate beneficial metabolic enzymes and transcriptional regulators ${ }^{34}$. Lipid droplets have also been found to act as innate immune hubs that can kill pathogens ${ }^{92}$. In contrast, lipid droplets also accumulate during old age and disease $\mathrm{e}^{93}$ and are detrimental in many contexts ${ }^{25-27,31,33,94-96}$. Furthermore, conditions that lead to fat storage but also lipolysis have been associated with longevity ${ }^{42,97-114}$. Our results may point to conditions (i.e. intact peroxisome function) that are needed for a high lipid droplet number to be beneficial for health.

We find that lipid droplet numbers at two different adult ages are predictive of remaining lifespan. Increased levels of reactive oxygen species during early development are also predictive of remaining lifespan, in part by reducing chromatin marks (e.g. those deposited by ASH-2) $)^{59}$. Early life events may also act determine lifespan trajectories by modulating MUFAs and rely on an intact lipid droplet-peroxisome network. This result is also intriguing in light of the observation that among dietary restricted individuals (in mice or humans) ${ }^{115,116}$, the fattest ones are the longest lived.

Our data reveal a functional connection between lipid droplets and peroxisomes in longevity. While physical interactions have been identified between lipid droplets and peroxisomes $^{65,69}$, a functional connection - especially in the context of aging - was not known. This hub between lipid droplets and peroxisomes appears to involve specific lipid - ether lipids and it may be critical not only in aging, but also in other biological processes or diseases. 
Finally, we also show for the first time that ether lipids regulate longevity, in part by modulating ferroptosis - a conserved form of cell death ${ }^{16,89,90}$. MUFAs have been found to protect from ferroptosis by displacing PUFAs from membrane lipids in mammalian cells ${ }^{117}$. It is possible that a high MUFA diet changes the balance among ether lipids in cells and prevent nonapoptotic forms of cell death in the organism. Given the conservation of lipid metabolism and organelles in all species, our findings open new avenues - based on lipid metabolism and composition - for promoting longevity and health.

\section{Methods}

\section{C. elegans and bacteria strains}

All C. elegans strains (N2 wild-type and mutants) used in this study are listed in Extended Data Table 4. Worms from mutant deletion strains were genotyped by PCR, and PCR amplicon sizes were used to check for deletions. C. elegans were grown and maintained at $20^{\circ} \mathrm{C}$ on standard Nematode Growth Media (NGM) plates seeded with a lawn of OP50-1 (gift from Dr. M.-W. Tan). For all experiments, worms were grown at $20^{\circ} \mathrm{C}$ on RNAi plates seeded with the RNAi strains HT115 (DE3) or the RNAi-sensitized iOP50 (gift from Dr. M. Wang) for ferroptosis induction by $g p x-1$ deletion. Some strains were provided by the CGC, which is funded by NIH Office of Research Infrastructure Programs (P40 OD010440) and the Mitani Laboratory at the Tokyo Women's Medical University School of Medicine.

The WBM1177 strain, which expresses a peroxisome localization sequence (SKL) fused to GFP driven by the eft-3 promoter, was generated by microinjection using CRISPR into WBM1140 (wbmIs65 [eft-3p::3XFLAG::dpy-10 crRNA::unc-54 3'UTR]) using the SKI LODGE system ${ }^{118}$. This system allows for knock-in of a single-copy of the construct downstream of the eft-3 promoter, which drives gene expression in all somatic cells and allows for ubiquitous expression of peroxisome-targeted GFP.

\section{RNA interference}

For knockdown by RNA interference (RNAi), worms were fed HT115 (DE3) (and in a few cases iOP50 bacteria) transformed with vectors expressing double-stranded RNA against the gene of interest. The bacteria HT115 strains expressing RNAi against the gene of interest were obtained 
from the Ahringer library (gift from Dr. A. Fire). RNAi clones were confirmed by sequencing. Experiments were performed on HT115 unless noted otherwise.

To generate RNAi-expressing bacteria, a single bacterial colony was used to inoculate LB containing $100 \mu \mathrm{g} / \mathrm{ml}$ ampicillin (Sigma Aldrich). The bacterial culture was grown overnight at $37^{\circ} \mathrm{C}$ to stationary phase and the expression of the double stranded RNAi was induced with 0.4 mM IPTG (Thermo Fisher) for $4 \mathrm{~h}$ at $37^{\circ} \mathrm{C}$. The cultures were centrifuged, and concentrated $30 \mathrm{x}$ by resuspending the bacterial pellet in $\mathrm{LB}_{\mathrm{Amp} / \mathrm{IPTG}}$ (LB with $100 \mu \mathrm{g} / \mathrm{ml}$ ampicillin, $0.4 \mathrm{mM}$ IPTG) at 1:30 of the initial volume. This bacterial resuspension was stored at $4^{\circ} \mathrm{C}$ for no longer than two weeks. Concentrated bacteria were added to $6-\mathrm{cm}$ or $10-\mathrm{cm}$ agar plates/ $\mathrm{KPO}_{4}$ containing 100 $\mu \mathrm{g} / \mathrm{ml}$ ampicillin and $0.4 \mathrm{mM}$ IPTG (RNAi plates). For knockdown experiments, worms were kept on 6-cm plates seeded with RNAi-expressing bacteria. Unless indicated otherwise, worms were fed the RNAi from egg lay on. For lpin-1 and fat-2, the RNAi was initiated at adult day one (young adult) to minimize effects of these gene knockdowns on development. For a negative control, worms were fed the empty-vector (L4440) bacteria strain. When 2 genes were knocked down simultaneously, the $\mathrm{OD}_{600}$ of the bacteria was adjusted to the same value $\left(\mathrm{OD}_{600}=50\right.$, stationary phase) and the RNAi expressing bacteria were mixed in a 1:1 ratio. To assess the gene knockdown efficiency in the context of single versus double RNAi, we performed real time quantitative PCR (RT-qPCR) with primers to each gene of interest (Extended Data Figure 1k), as indicated below in the "Real time quantitative PCR" section.

\section{Real time quantitative PCR}

To test the efficiency of gene knockdown in the context of single versus double RNAi, quantitative RT-PCR was performed as described ${ }^{6}$. Briefly, 300 worms were subjected to RNAiexpressing bacteria (or control bacteria) on 6-cm RNAi plates. For experiments with lpin-1 RNAi, knockdown for all conditions was initiated at young adult age and worms were harvested at adult day 2. Worms were washed 3 times with $\mathrm{M} 9$ buffer $\left(22 \mathrm{mM} \mathrm{KH}_{2} \mathrm{PO}_{4}, 34 \mathrm{mM} \mathrm{K}_{2} \mathrm{HPO}_{4}\right.$, $86 \mathrm{mM} \mathrm{NaCl}_{1} 1 \mathrm{mM} \mathrm{MgSO}_{4}$ ) to remove residual bacteria in the worm pellet. To isolate total RNA, worm pellets were resuspended in $500 \mu 1$ Trizol Reagent (Invitrogen) and subjected to six freeze-thaw cycles in a dry ice-ethanol bath. RNA was extracted according to the standard Trizol procedure, resuspended in $30 \mu \mathrm{l}$ of RNase- and DNase- free water, and quantified using the Nanodrop (Thermo Fisher). RNA was treated with DNAse (Thermo Fisher, Cat\#:18068015), 
followed by reverse-transcription using Oligo (dT) 20 primers (Invitrogen, 18418020) and

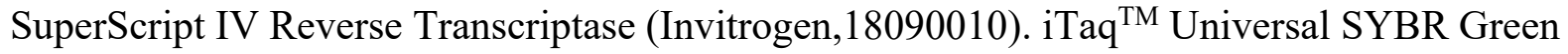
super mix (Bio-Rad,1725124) was used in a $20 \mu \mathrm{l}$ reaction volume for the real time quantitative PCR reaction. Primers were designed to span exon-exon junctions and used at a final concentration of $250 \mathrm{nM}$. All primers are listed in Extended Data Table 5. Real time quantitative PCR was performed using the $\mathrm{C} 1000$ thermal cycler (Bio-Rad). Melt curves were examined to ensure specificity of qPCR primers. Results were analyzed using the $\Delta \Delta$-CT method. For each biological replicate, the median Ct value of 3 technical replicates was analyzed and act-1 served as the internal reference gene. Bar plots were plotted using Prism 8. Experiment was performed twice independently, with 3 replicates each. Statistical significance was tested using the unpaired, non-parametric Mann-Whitney test.

\section{Scattered Raman Spectroscopy for lipid droplet quantification}

To visualize all lipids in a label-free way, we used Stimulated Raman Scattering (SRS) microscopy ${ }^{35}$. Individual worms (treated with control or RNAi-expressing HT115 bacteria) were imaged post reproduction at adult day 6 (middle-age). Worms were mounted on a $2 \%$ agar pad, anesthetized with $50 \mathrm{mM}$ sodium azide and covered with a glass cover slide for imaging. Worms were imaged directly after mounting to avoid confounds from starvation on the microscope slide using an SRS setup as described previously ${ }^{119}$. The experimental set-up was built on an inverted microscope (IX81, Olympus, Shinjuku, Japan). For SRS microscopy, spatially and temporally overlapped pulsed Pump (tunable from 720 to 990 nm, 7 ps, 80 MHz repetition rate) and Stokes (1064 nm, $5 \sim 6 \mathrm{ps}, 80 \mathrm{MHz}$ repetition rate, modulated at $8 \mathrm{MHz}$ ) beams provided by picoEMERALD (Applied Physics \& Electronics, Berlin, Germany) were coupled into an inverted laser-scanning microscope (FV1000 MPE; Olympus) optimized for near-IR throughput. A $20 \times$ air objective (UPlanSAPO; 0.75N.A.; Olymp) and a 60x water objective (UPlanAPO/IR; 1.2N.A.; Olympus) were used for imaging. After passing through the sample, the forward going Pump and Stokes beams were collected in transmission by an air condenser (0.9 N.A., Olympus) for the 20x magnification and an oil condenser (1.4 N.A., Olympus) for the 60x magnification. A high OD bandpass filter (890/220, Chroma, Bellows Falls, VT) was used to block the Stokes beam completely and to transmit only the Pump beam onto a large area Si photodiode (FDS1010, Thorlabs) to for the detection of the stimulated Raman loss signal. The output current from the photodiode was terminated, filtered, and demodulated by a lock-in amplifier (HF2LI; Zurich 
Instruments, Zurich, Switzerland) at $8 \mathrm{MHz}$ to ensure shot noise-limited detection sensitivity. The laser power of IR and OPO were set at $600 \mathrm{~mW}$. For lipid imaging, $\mathrm{CH}_{2}$ signals from lipid droplets were imaged at $2845 \mathrm{~cm}^{-1}$ in SRS channel. These settings are used to visualize all lipids, as they are rich in these type of bonds. For lipid droplet imaging, one experiment was recorded at 60x and the duplicate was recorded using a 20x objective. Within one replicate, the same threshold was applied to all images and lipid droplets were quantified in a $26 \times 26 \mu \mathrm{m}^{2}$ area using the analyze particle function in Fiji version 2.0.0 ( $\left.\operatorname{Ref}^{120}\right)$. Experiments were not performed in a blinded manner. For each experiment, $n>18$ hermaphrodites were imaged per conditions, and the experiment was carried out in 2 independent experiments. Lipid droplet numbers and intensities were plotted using dot plots in Prism 8 and statistically significant differences between samples were assessed using the unpaired, non-parametric Mann-Whitney test.

\section{Confocal microscopy for lipid droplet quantification}

To visualize lipid droplets by confocal microscopy, we used a reporter strain expressing the lipid droplet protein DHS-3 fused to green fluorescent protein (GFP), which has been previously characterized as a lipid droplet marker ${ }^{40,41}$. Transgenic LIU1 (dhs-3p::DHS-3::GFP) worms were imaged post reproduction at adult day 6, unless noted otherwise. For each experiment, $\sim 20$ worms were imaged per condition. Worms were mounted on a 2\% agar pad, anesthetized with 50 $\mathrm{mM}$ sodium azide and covered with a glass cover slide for imaging. Worms were imaged with the Nikon Eclipse Ti confocal microscope with a Zyla sCMOS camera (Andor) and NISElements software (AR 4.30.062, 64 bit) using the 100x oil objective (Nikon, Plan Apo, 100x) or a Zeiss confocal microscope (LSM900, Axio Observer) using the 63x oil objective (PlanApochromat) and Zen software (3.0, blue). The mid-intestinal region was imaged in $0.2 \mu \mathrm{m}$ slices and all images were taken using the same exposure time. Lipid droplet numbers were analyzed in Fiji version 2.0.0 ( $\operatorname{Ref}^{120}$ ) by generating $\mathrm{z}$-stack projections of 5 slices, applying the same threshold to all images and manually counting the lipid droplets in a $26 \times 26 \mu \mathrm{m}^{2}$ area. Lipid droplet diameters were analyzed in Fiji version 2.0.0 ( $\operatorname{Ref}^{120}$ ) by generating $\mathrm{z}$-stack projections of 5 slices, applying the same threshold to all images and manually measuring the diameter of all lipid droplets in focus. 
To measure lipid droplets in males, transgenic LIU1 (dhs-3p::DHS-3::GFP) males were generated by a brief $1 \mathrm{~h}$ heat shock of L4 hermaphrodites at $36^{\circ} \mathrm{C}$. The male progeny were picked and maintained by crossing them with hermaphrodites. For all other steps, male worms were processed similarly as described above.

To measure hypodermal lipid droplets, transgenic LIU2 (plin-1p::PLIN-1::mCherry) worms were imaged post reproduction at adult day 6 . This reporter strain has been used to visualize hypodermal and intestinal lipid droplets ${ }^{40}$. For each experiment, $\sim 20$ worms were imaged per condition. Worms were processed similarly as described above with the following differences. To analyze hypodermal lipid droplets, the head hypodermis was imaged to avoid accidently imaging intestinal lipid droplets. As the width of the worms is smaller in the head region, a smaller area $15 \times 15$ area $\mu \mathrm{m}^{2}$ was selected to count the number of lipid droplets.

To visualize lipid droplets in eggs, nile red staining was performed as described before ${ }^{41}$. Briefly, adult day 1 hermaphrodites were fixed in $40 \%$ isopropanol for 3 minutes and stained with $8 \mu \mathrm{M}$ nile red (MP biomedicals \# 0215174450) for 2 hours in the dark. Worms were mounted and imaged similarly as described before. One to two fertilized eggs per worm were imaged in utero in $0.2 \mu \mathrm{m}$ slices. For each experiment, $\sim 20$ worms were imaged per condition. The number of lipid droplets was counted in a $16 \times 16 \mu \mathrm{m}^{2}$ area using the Squassh plugin ${ }^{121}$. The following plugin settings were used and kept consistent across experiments. The background was subtracted with a rolling ball radius of 10 pixels, the segmentation parameters were set at 0.005 with a minimum object intensity of 0.15 .

All experiments and lipid droplet analysis were performed in a blinded manner. Each experiment was at least carried out twice independently. Lipid droplet numbers and intensities were plotted using dot plots in Prism 8 and statistically significant differences between samples were assessed using the unpaired, non-parametric Mann-Whitney test.

\section{Monounsaturated fatty acid supplementation}

To increase the level of specific fatty acids in C. elegans, we performed dietary supplementation as described before ${ }^{6}$. In short, fatty acid sodium derivatives (Nu-check prep) of oleic acid (C18:1n9 cis) and elaidic acid (C18:1n9 trans) were dissolved in water at $100 \mathrm{mM}$. To dissolve elaidic acid, the solution was heated in a water bath to $50^{\circ} \mathrm{C}$ for no more than 10 minutes. The detergent Tergitol (NP-40, Sigma Aldrich) was added at a concentration of $0.001 \%$ to RNAi agar 
plates prior to autoclaving. After autoclaving, the agar media plates were cooled down to approximately $60^{\circ} \mathrm{C}$ and the fatty acids solutions were added to a final concentration of $0.8 \mathrm{mM}$. The agar media was stirred for 2 minutes after addition of the fatty acids to ensure even distribution. The plates were dried overnight in a dark ventilates space, stored at $4^{\circ} \mathrm{C}$ and used within a month. Bacteria were seeded on oleic acid or elaidic acid plates 24 hours before worms were transferred to the plates. For supplementation experiments, worms were kept on oleic acid or elaidic acid plates from egg lay on. For experiments including lpin-1 knock-down, RNAi and fatty acid supplementation were both started simultaneously at adult day 1 because of the negative effect of lpin-1 knock-down on development.

\section{Lifespan assays}

All C. elegans lifespan assays were performed at $20^{\circ} \mathrm{C}$ on RNAi plates. Hermaphrodites were age-synchronized with a short 4 hour egg lay using synchronized young (adult day 1) parents. Hermaphrodites were transferred to new plates and scored at least every other day to avoid the presence of confounding progeny. Each lifespan assay was performed with 3 plates of $\sim 30$ worms per 6-cm RNAi plates ( $\sim 90$ worms total). Worms were scored as censored if they crawled off the media or if they died upon vulval rupture/internal hatching. Worms were scored as dead when they failed to move upon gentle prodding with a platinum wire pick ( $90 \% \mathrm{Pt}, 10 \% \mathrm{Ir})$. For lifespan curves, censored worms were included until the day of censorship. All lifespan experiments were performed in a blinded and randomized manner. Each experiment was carried out at least twice independently. Kaplan-Meier survival curves were plotted in Prism 8. For pairwise comparison, the log-rank (Mantel-Cox) statistical test in Prism was used. To test if two interventions significantly interact with each other, the Cox proportional hazard test was applied using R (Version 3.6.3). For all lifespan statistics, see Extended Data Table 1.

\section{Gas chromatography/mass spectrometry analysis of fatty acid profiles}

To ensure that fatty acid profiles change upon ash-2 RNAi, targeted gas chromatography/mass spectrometry (GC/MS) was performed as described before ${ }^{6}$. Briefly, for each condition, approximately 500 age-synchronized adult day 1 animals were collected in M9 buffer and washed 3 times to remove residual bacteria in the worm pellets. Worm pellets were lyzed by sonication and protein concentration of the lysate was determined using the Pierce BCA Protein Assay Kit (Thermo- Scientific). The fatty acid C13:0 (NuChek Prep, dissolved in methanol), was 
added to each sample to serve as the internal reference control for variations introduced during derivatization and extraction steps. Fatty acids were derivatized into their respective fatty acid methyl ester (FAME) by incubation in $2 \% \mathrm{H}_{2} \mathrm{SO}_{4}$ (Sigma Aldrich) in methanol (Fisher) at $55^{\circ} \mathrm{C}$ overnight. The reaction was stopped by the addition of $1.5 \mathrm{ml}$ water (Fisher, MS grade). FAMEs were extracted in a $300 \mu 1$ hexane (Sigma Aldrich) by vigorous vortexing and centrifuging at $1000 \mathrm{rpm}$ for 1 minute. The hexane layer, containing the FAMEs, was transferred into an amber GC vial (Agilent). FAME analysis was performed using an Agilent 7890A gas chromatograph equipped with an HP-5MS column. Each FAME peak was identified based on its retention time and unique fragmentation ions and quantified using a serial dilution standard curve.

FAME abundance measured by GC-MS was normalized to the internal C13:0 reference control of each sample. For each sample FAMEs concentration $(\mu \mathrm{g} / \mathrm{ml})$ was normalized to protein concentration $(\mathrm{mg} / \mathrm{ml})$ as microgram of fatty acid detected per milligram of protein $(\mu \mathrm{g} / \mathrm{mg})$. The fatty acid concentration ( $\mu \mathrm{g} / \mathrm{mg}$ ) for $a s h-2$ RNAi was normalized to the fatty acid concentration $(\mu \mathrm{g} / \mathrm{mg})$ of the empty vector control. The final ratio is expressed as relative fatty acid levels in the graph. Each experiment was at least carried out twice independently. Relative fatty acid abundances were plotted Prism 8 and statistically significant differences between samples were assessed using the unpaired, non-parametric Mann Whitney test with Benjamini and Hochberg test for multiple hypothesis correction.

\section{Separating fluorescent worms using the BioSorter}

To sort worms according to their lipid droplet number at young age (adult day 1), we used the large particle BioSorter (Union Biometrica). To retrieve a large number of age-synchronized worms for the sorting procedure, approximately 5,000 eggs were laid by age-synchronized adult day 1 parent worms expressing DHS-3 fused to GFP ( $d h s-3 p:$ DHS-3::GFP, LIU1). After approximately 72 hours at $20^{\circ} \mathrm{C}$, the laid eggs developed into adult worms that were collected in M9 buffer (22 mM KH $\left.2 \mathrm{PO}_{4}, 34 \mathrm{mM} \mathrm{K}_{2} \mathrm{HPO}_{4}, 86 \mathrm{mM} \mathrm{NaCl}_{1} 1 \mathrm{mM} \mathrm{MgSO}_{4}\right)$ and sorted on the large particle flow cytometer BioSorter (Union Biometrica) with a 6.5 psi sample cup pressure, 2.00 psi diverter pressure, $50 \%$ sheath flow rate, rotary valve, $8.0 \mathrm{~ms}$ drop width and $10.0 \mathrm{~ms}$ sort delay using the FlowPilot III software. Using a dissecting microscope, we visually confirmed these settings enabled us to sort the accurate number of worms. The highest and lowest 10\% fluorescent worms of the population were sorted using the $488 \mathrm{~nm}$ laser (Extended Data Fig. 2c). Worms were directly sorted onto 6-cm RNAi plates containing a bacterial lawn 
441 (HT115, empty vector). We confirmed that the sorting reflects the lipid droplet number by

442 fluorescence confocal microscopy (Fig. 2d, e). For this, worms were mounted on a 2\% agar pad,

443 anesthetized with $50 \mathrm{mM}$ sodium azide and covered with a glass cover slide for imaging. Worms

444 were imaged with the Nikon Eclipse Ti confocal microscope with a Zyla sCMOS camera

445 (Andor) and NIS-Elements software (AR 4.30.062, 64 bit) using the 100x oil objective (Nikon,

446 Plan Apo, 100x) or a Zeiss confocal microscope (LSM900, Axio Observer) using the 63x oil

447

448

449

450

451

452

453

454

455

456

457

458

459

460

461

462

463

464

465

466

467

468

469

470

471

472

objective (Plan-Apochromat) and Zen software (3.0, blue). Lipid droplet quantification was performed blinded and as described above. Each experiment was performed at least twice independently. BioSorter graphs were plotted using the FlowPilot III software, and confocal microscopy quantifications were plotted in Prism 8. Statistically significant differences between samples were assessed using the unpaired, non-parametric Mann-Whitney test.

\section{Separating fluorescent worms using manual sorting}

To sort worms according to their fluorescence at middle age, we manually separated high and low fluorescent worms on a fluorescent dissecting microscope. To retrieve a large number of age-synchronized worms for the sorting procedure, approximately 5,000 eggs were laid by agesynchronized adult day 1 parent worms ( $d h s-3 p:: D H S-3:: G F P$, LIU1). After approximately 72 hours at $20^{\circ} \mathrm{C}$, the eggs developed into adult worms. The adult worms were washed each day during their reproductive period to separate the adult worms from larvae/eggs. For this, worms were collected in M9 buffer $\left(22 \mathrm{mM} \mathrm{KH}_{2} \mathrm{PO}_{4}, 34 \mathrm{mM} \mathrm{K}_{2} \mathrm{HPO}_{4}, 86 \mathrm{mM} \mathrm{NaCl}, 1 \mathrm{mM} \mathrm{MgSO}_{4}\right)$ in a $15 \mathrm{ml}$ tube and allowed to settle to the bottom of the tube. The supernatant was removed and worms were resuspended in $15 \mathrm{ml}$ of fresh M9. This washing procedure was repeated 5 times and the adult worms were transferred to fresh $10 \mathrm{~cm}$ RNAi plates seeded with $1 \mathrm{ml}$ empty vector HT115 bacteria. Post-reproductive middle-aged worms (adult day 6, no noticeable egg production) were sorted under a fluorescent dissecting microscope (Leica M165 FC) equipped with a Sola light engine (Leica) on 6-cm RNAi plates containing a bacterial lawn (HT115, empty vector). We confirmed that the sorting reflects the lipid droplet number by fluorescence confocal microscopy (Fig. 2g). Worms were mounted on a 2\% agar pad, anesthetized with $50 \mathrm{mM}$ sodium azide and covered with a glass cover slide for imaging. Worms were imaged with the Nikon Eclipse Ti confocal microscope with a Zyla sCMOS camera (Andor) and NIS-Elements software (AR 4.30.062, 64 bit) using the 100x oil objective (Nikon, Plan Apo, 100x) or a Zeiss confocal microscope (LSM900, Axio Observer) using the 63x oil objective (Plan-Apochromat) and Zen 
473 software (3.0, blue). Lipid droplet quantification was performed blinded and as described above.

474 Each experiment was performed at least twice independently. Graphs were plotted using dot

475 plots in Prism 8. Statistically significant differences between samples were assessed using the

476

477

478

479

480

481

482

483

484

485

486

487

488

489

490

491

492

493

494

495

496

497

498

499

500

501

502

503

504 unpaired, non-parametric Mann-Whitney test.

\section{Re-analysis of gene expression datasets}

We re-analyzed two independent gene expression datasets that were generated previously in the lab, and we tested GO term enrichment in conditions that lead to MUFA accumulation. First, we analyzed a RNA-seq dataset from the intestine of adult day 1 worms treated with ash-2 RNAi ${ }^{6}$. We selected the genes that had a $\log 2$ fold-enrichment larger than 1 (42 genes/adjusted $P$ value $<0.05$ ) and analyzed enrichment for GO terms using WormEnrichR ${ }^{122,123}$. All categories of GO terms were included in subsequent analysis. Second, we analyzed a microarray dataset from the entire body of post-reproductive worms treated with ash-2 $\mathrm{RNAi}^{5}$. We selected the genes that were had a $\log 2$ fold-enrichment larger than 1 (304 genes/adjusted p-value $<0.05)$ and analyzed enrichment for GO terms using WormEnrichR for GO terms. All categories of GO terms were included in subsequent analysis. Significantly enriched GO terms (combined score $>5)^{122-124}$ that were shared between the two datasets were plotted using R (Version 3.6.3). Duplicated GO terms were deleted based on the higher combined score in the RNAseq dataset. The combined score was calculated by multiplying the $P$-value retrieved from the Fisher's Exact test with the z-score. For detailed list of all GO terms, see Extended Data Table 2.

\section{Confocal microscopy for peroxisome quantification}

Peroxisomes were visualized by confocal microscopy using worm strains that express a fluorophore fused to a peroxisome localization sequence (SKL). The fluorophore was either GFP for ges-1p::GFP-SKL(VS15) and eft-3p::GFP-SKL (WBM1177) or mRFP for vha-6p::mRFPSKL; $d s h-3 p:: D H S-3: \because G F P(A B R 161)$. Transgenic worms were imaged post-reproduction at adult day 6 , unless noted otherwise. The last intestinal cell was imaged in a similar way as described before ${ }^{66}$. We used these cells for quantification because they retain homogenous fluorophore expression with age. Worms were mounted on a $\%$ agar pad, anesthetized with 50 $\mathrm{mM}$ sodium azide and covered with a glass cover slide for imaging. Worms were imaged with the Nikon Eclipse Ti confocal microscope with a Zyla sCMOS camera (Andor) and NISElements software (AR 4.30.062, 64 bit) using the 100x objective or a Zeiss confocal 
microscope (LSM900, Axio Observer) using the 63x oil objective (Plan-Apochromat) and Zen software (3.0, blue). The last intestinal cell was imaged in $0.2 \mu \mathrm{m}$ slices and all images were taken using the same exposure time. Images were analyzed in Fiji version 2.0.0 ( $\left.\operatorname{Ref}^{120}\right)$ by generating z-stack projections of 5 slices, applying the same threshold to all images and automatically counting the lipid droplets in a $26 \times 26 \mu \mathrm{m}^{2}$ area using the Squassh plugin ${ }^{121}$. This plugin was used to count the numbers of particles and analyze their fluorescence intensity. The following settings were used and kept consistent across experiments. The background was subtracted with a rolling ball radius of 10 , the segmentation parameters were set at 0.005 with a minimum object intensity of 0.15 . Peroxisomes usually appeared as fluorescent puncta. Rarely, when the fluorophore was only cytosolic and failed to localize to peroxisomes, the plugin did not perform correctly, and these quantifications were excluded from further analysis. All experiments and analysis were performed in a blinded manner unless noted otherwise in the Source Data Tables. For each experiment, 20 worms were imaged per condition. Each experiment was performed at least twice independently. Peroxisome numbers were plotted using dot plots in Prism 8 and statistically significant differences between samples were tested using the unpaired, non-parametric Mann-Whitney test.

\section{Lipid droplet and peroxisome number as a function of age}

To visualize lipid droplet and peroxisome dynamics as a function of age, we imaged worms during their adult life. Lipid droplets and peroxisomes were visualized by confocal microscopy at different ages and quantified as described above. Peroxisomes were visualized by mRFP and lipid droplets by GFP in the vha-6p::mRFP-SKL; $d s h-3 p:: D H S-3:: G F P(\mathrm{ABR} 161)$ double marker strain as described above. The last intestinal cells were imaged as described above. Lipid droplet and peroxisome numbers were quantified using the same region of interest per worm. All experiments and analysis were performed in a blinded manner. For each experiment, $\sim 24$ worms were imaged per condition. Experiments carried out twice independently. Mean organelle numbers and regression lines were plotted using Prism 8.

\section{Targeted lipid droplet and peroxisome screen}

To analyze how lipid droplet genes influence peroxisome numbers and vice versa, we performed a targeted RNAi screen. We selected genes to target for the screen based on proteins identified by mass spectrometry at the surface of lipid droplets ${ }^{40,41}$ and based on annotated protein function 
in lipid metabolism, lipid droplet biology, and peroxisome biology. As a positive control, we used ash-2 RNAi and prx-5 RNAi. We assessed the effect of RNAi knock-down of these genes on lipid droplet and peroxisome numbers at day 6 of life in approximately 30 animals per condition. Lipid droplets and peroxisomes were visualized by confocal microscopy and quantified as described above. The screen was performed in six groups. Each group contained the empty vector control, ash-2 and prx-5 RNAi. Peroxisomes were visualized by mRFP (except for the first group) and lipid droplets by GFP in the vha-6p::mRFP-SKL; $d s h-3 p:: D H S-3:: G F P$ (ABR161) double-marker strain. For the first group, peroxisomes were measured in another set of worms, using the single marker strain ges-1p::GFP-SKL (VS15) and the corresponding lipid droplet numbers were from an independent experiment. For all other groups, lipid droplet and peroxisome numbers were quantified using the same $26 \times 26 \mu \mathrm{m}^{2}$ area region of interest per worm in the last intestinal cell. Organelle numbers were normalized to the control empty vector RNAi within the corresponding group. Lipid droplet numbers were quantified manually and peroxisome numbers were quantified using the Squassh plugin ${ }^{121}$ as described above. Peroxisomes usually appear as puncta. Rarely, when the fluorophore was only cytosolic and failed to localize to peroxisomes, the plugin did not perform correctly, and these quantifications were excluded from further analysis. All experiments and analysis were performed in a blinded manner. To assess correlation of organelle numbers, the mean organelle number normalized to the control condition (empty vector RNAi) was plotted using Prism and statistically significant correlation was tested using the Pearson's $r$ test. Manhattan graphs were plotted using Prism8 and statistical significance was tested using a two-tailed Mann-Whitney test and BenjaminiHochberg correction was used for multiple hypothesis correction.

\section{Sample preparation for lipidomics}

To analyze lipid composition using mass spectrometry, hermaphrodites were treated with control (empty vector) RNAi, prx-5 RNAi, ash-2 RNAi, and prx-5/ash-2 double RNAi until middle-age (adult day 6). Each condition consists of six biological replicates. To retrieve a large number of age-synchronized worms for the procedure, approximately 500 eggs were laid by agesynchronized adult day 1 wildtype parents per replicate plate. After 2 hours of egg laying, the parents were removed and the plates were checked that no parents remained. Once the worms reached the young adult stage, they were washed each day to separate the adult worms from larvae/eggs. For this, worms were collected in M9 buffer $\left(22 \mathrm{mM} \mathrm{KH}_{2} \mathrm{PO}_{4}, 34 \mathrm{mM} \mathrm{K}_{2} \mathrm{HPO}_{4}, 86\right.$ 
$\mathrm{mM} \mathrm{NaCl}, 1 \mathrm{mM} \mathrm{MgSO}_{4}$ ) and allowed to settle to the bottom of the tube. The supernatant was removed and fresh M9 was added. This washing procedure was repeated 6 times and the adult worms were transferred to fresh 6-cm RNAi plates seeded with $500 \mu 1$ RNAi-expressing HT115 bacteria. At middle-age (adult day 6), worms were transferred to empty RNAi plates without any bacteria for 15 minutes, to clear residual bacteria in the gut. Worms were then collected in $200 \mu 1$ M9 in protein-low bind Eppendorf tubes (cat \# 13-698-794). Worms were lyzed using a prechilled stainless-steel homogenizer (Wheaton, cat \# 357572) and were homogenized with 10 plunger strokes. The lysate (from approximately 500 worms) was frozen on dry ice and stored at $-80^{\circ} \mathrm{C}$.

\section{Lipid isolation for mass spectrometry}

Lipids from the whole worm lysates were extracted using a biphasic separation with methyl tertbutyl ether (MTBE), methanol and water ${ }^{125}$. All reagents used are for lipidomics are LC/MS grade. Briefly, $298 \mu \mathrm{l}$ of ice-cold methanol and $2 \mu \mathrm{l}$ of internal standard (equiSPLASH, Avanti Polar Lipids, cat\# 330731) were added to $50 \mu \mathrm{l}$ of worm lysate. The mixture was vortexed for 20 seconds and $1000 \mu \mathrm{l}$ of ice-cold MTBE was added. The mixture was incubated under agitation for 30 minutes at $4{ }^{\circ} \mathrm{C}$. After addition of $250 \mu$ of water, the samples were vortexed for 1 minute and centrifuged at 14,000 g for 10 minutes at room temperature. The upper phase containing the lipids was collected and dried down under nitrogen. The dry extracts were reconstituted with 300 $\mu \mathrm{l}$ of 9:1 methanol:toluene (Fisher Scientific) with $10 \mathrm{mM}$ of ammonium acetate (Sigma Aldrich) and centrifuged at $14,000 \mathrm{~g}$ for 5 minutes before analysis. Water extracted using the same protocol was used as a blank control. Samples were randomized in all cases during lipid extraction.

\section{Liquid chromatography mass spectrometry}

To identify complex lipids, isolated lipids were analyzed with untargeted lipidomics using liquid chromatography coupled to a Q Exactive mass spectrometer (Thermo Fisher Scientific) (LC/MS). Lipids were separated using an Accucore C30 column 2.1 x 150 mm, $2.6 \mu \mathrm{m}$ (Thermo Scientific, cat\# 27826-152130) and mobile phase solvents consisted in $1 \mathrm{mM}$ ammonium formate and $0.1 \%$ formic acid in $60 / 40$ acetonitrile/water (A) and $1 \mathrm{mM}$ ammonium formate and $0.1 \%$ formic acid in 90/10 isopropanol/acetonitrile (B). The gradient profile used was 30\% B for 3 minutes, 30-43\% B over 5 minutes, 43-50\% B over 1 minute, 55-90\% B over 9 minutes, 90- 
601

602

603

604

605

606

607

608

609

610

611

612

613

614

615

616

617

618

619

620

621

622

623

624

625

626

627

628

629

630

631

632

99\% B over 9 minutes and 99\% B for 5 minutes. Lipids were eluted from the column at 0.2 $\mathrm{ml} / \mathrm{min}$, the oven temperature was set at $30^{\circ} \mathrm{C}$, and the injection volume was $15 \mu \mathrm{l}$. Autosampler temperature was set at $15^{\circ} \mathrm{C}$ to prevent lipid aggregation. The Q Exactive plus was equipped with a HESI-II probe and operated in full MS scan mode for all the samples. MS/MS spectra were acquired in data-dependent acquisition mode on pooled samples. The source conditions were as follows: Spray Voltage $=3.5 \mathrm{kV}$ (ESI positive mode), Vaporizer $=200^{\circ} \mathrm{C}$, Capillary Temperature $=375^{\circ} \mathrm{C}, \mathrm{S}-L e n s=55.0 \%$, SheathGas $=40$, Auxiliary gas $=8$, SweepGas $=0$. The acquisition settings were as follows: $\mathrm{AGC}(\mathrm{MS})=3 \mathrm{e} 6, \mathrm{AGC}\left(\mathrm{MS}^{2}\right)=1 \mathrm{e}$, Maximum Injection Time $(\mathrm{MS})=200 \mathrm{~ms}$, Maximum Injection Time $\left(\mathrm{MS}^{2}\right)=50 \mathrm{~ms}$, Mass Range = 260-1,900 Da, Resolution MS = 70,000 (FWHM at m/z 200), Resolution $\mathrm{MS}^{2}=35,000(\mathrm{FWHM}$ at m/z 200), $\mathrm{MS}^{2}$ spectra was acquired in top-10 ions in each cycle, Isolation Window $=1.0 \mathrm{~m} / \mathrm{z}$, Dynamic Exclusion $=12 \mathrm{~s}$, Normalized Collision Energy $(\mathrm{NCE})=25-30$. External calibration was performed using an infusion of Pierce LTQ Velos ESI Positive Ion Calibration Solution.

\section{Analysis of mass spectrometry results}

Lipid species were identified by matching the precursor ion mass to a database and the experimental MS/MS spectra to a spectral library containing theoretical fragmentation spectra using LipidSearch software version 4.1 (Thermo Scientific) ${ }^{126}$. Further data processing was done using an in-house analysis pipeline written in $\mathrm{R}$ (Version 3.6.3, available in Github at https://github.com/brunetlab).

Briefly, processing for samples and spike-in standards were done in the same way. All ions for one lipid were aggregated and lipids with a signal $>0$ discarded from further analysis. Lipid species were quantified using the corresponding internal standard (equiSPLASH, Avanti Polar Lipids, cat\# 330731) for each lipid class. Lipids with signals lower than 3x blank signal were discarded. Lipids with more than $50 \%$ of missing values were discarded and for the remaining missing values, imputation was performed. For this, a value was randomly assigned based on the bottom 5\% for the corresponding lipid. Lipids were filtered for a coefficient of variance $<0.5$. Each sample was divided by its corresponding protein concentration to correct for sample input variations. To calculate normalized abundance, each lipid within a sample was divided by the sample median followed by multiplication with the global median. This resulted in a total of 500 filtered and normalized lipids belonging to 16 lipid classes. For a complete list of all lipidomic data, see Extended Data Table 6. Changes in the most abundant lipid classes - diacyglycerol 
633 (DG), phosphatidylethanolamine (PE), phosphatidylcholine (PC) and phosphatidylinositol (PI) -

634 as well as differences in abundance of fatty acids grouped by length are listed in Source Data

635 Extended Data Fig 4 and Extended Data Table 7. Together, these data indicate that peroxisome

636

637

638

639

640

641

642

643

644

645

646

647

648

649

650

651

652

653

654

655

656

657

658

659

660

661

662

663

664 deficiency widely remodels the lipidome, with accumulation of long-chain fatty acids, in agreement with a previously published study ${ }^{127}$. We also observed an increase in saturated fatty acids upon peroxisome deficiency (Fig. 4b), consistent with previous studies that detected an increase in saturated long-chain fatty acids upon peroxisome deficiency ${ }^{128,129}$. Box plots were plotted using R (Version 3.6.3). Statistical significance was tested using a two-tailed MannWhitney test and Benjamini-Hochberg correction was used for multiple hypothesis correction. To test if two interventions significantly interact with each other, a two-way ANOVA was applied using Prism8.

\section{Role of ferroptosis in longevity by MUFAs and lipid droplets}

To test the effect of ferroptosis - a non-apoptic form of cell death involving oxidized lipids - on lifespan, we modulated the ferroptosis process in C. elegans. To induce ferroptosis, we performed RNAi knock-down of the $g p x-1$ gene, which encodes a glutathione peroxidase. As ferroptosis induction was shown to be enhanced on an OP50 diet (compared to an HT115 diet $)^{15,130}$, we fed $g p x-1(\operatorname{tm} 2100)$ mutants with iOP50 bacteria ${ }^{131}$ expressing ash-2 and control empty vector (L4440) RNAi. To inhibit ferroptosis, we exposed worms to Salinazid (LGC Standards/Dr. Ehrenstorfer cat \# DRE-C16904350), a lipophilic compound that scavenges intracellular iron, thereby inhibiting iron-triggered lipid peroxidation that induce ferroptosis ${ }^{88,132}$. Salinazid was dissolved in DMSO and added to autoclaved RNAi media before solidification to a final concentration of $250 \mu \mathrm{M}$. Plates were dried overnight in a dark ventilated space, stored at $4^{\circ} \mathrm{C}$, and used within a month. Worms were transferred to Salinazid and control (DMSO) plates, at adult day 1. Lifespan was measured as described above.

\section{Statistics}

No statistical method was used to predetermine sample size. Parameters such as the minimum n value, mean \pm standard deviation and significant $P$-values are reported in the figures, the figure legends or the corresponding source files. Significance was defined by a $P$-value $<0.05$. All $P$ values can be found in the source data tables. Pairwise comparisons were made using the Mann Whitney test (also called Wilcoxon rank sum test) in Prism8. When over five comparisons were 
665

666

667

668

669

670

671

672

673

674

675

676

677

678

679

680

681

682

683

684

685

686

687

688

689

690

691

692

693

made, the Benjamini-Hochberg correction was used for multiple hypothesis correction. When testing for correlation, the Pearson correlation test was used and normality distribution was confirmed using the Kolmogorov-Smirnov test using Prism8. To test if two interventions interact with each other, a two-way ANOVA was applied using Prism8. For pairwise comparison of lifespan data, the log-rank (Mantel-Cox) statistical test in Prism8 was used. To test if two lifespan interventions significantly interact with each other, the Cox proportional hazard test was applied using R (Version 3.6.3) which was reported in the figure legends. All lifespan statistics are reported in Extended Data Table S1. Statistical analyses were performed using Prism8 or R (Version 3.6.3).

\section{Acknowledgements}

We thank the Stanford Mass Spectrometry facility (Vincent Coates Foundation Mass Spectrometry Laboratory) and Dr. Allis Chien for GC/MS use and support. We thank the Stanford High Throughput BioCenter and Dr. David E. Solow-Cordero for BioSorter use and support (supported by NIH SIG S10OD025004). We thank the Brunet Lab, and in particular Drs. Lauren N. Booth, Paloma Navarro and Xiaoai Zhao for their feedback and reading of the manuscript. We thank Drs. Andrew S. McKay and Xiaoai Zhao for independently checking scripts used in this study. We thank Drs. Jonathan Long and Scott Dixon for insightful discussion and for critical reading of the manuscript. This work was supported by R01AG054201 (A.B., W.B.M.), a DFG German Research Foundation postdoctoral fellowship (K.P.), and a Hillblom Foundation postdoctoral fellowship (J.W.M.).

\section{Authors contributions}

K.P. and A.B. planned the study. K.P. performed and analyzed all experiments, except for those indicated below. A.H. performed and analyzed the peroxisome quantification, targeted screen, independent repeats of lifespan experiments, and Salinazid experiments. J.W.M. performed the peroxisome sorting experiment and participated in the planning of the study. M.C. ran and analyzed the lipidomics samples supervised by M.P.S. L.M.M. and E.D.L. helped K.P. with data collection on lipid droplet and peroxisome number, respectively. Y.Y. performed and analyzed 
the SRS experiments supervised by M.C.W. C.G.G. generated several C. elegans lines and P.Y. generated the WBM1177 line supervised by W.B.M., and C.G.G. and W.B.M. also had intellectual input in the study. K.P. and A.B. wrote the manuscript, and all authors provided comments.

\section{Competing Interests}

The authors declare no competing interests.

\section{Data availability}

All data generated or analyzed during this study are included in this published article (and its supplementary information files). All lipidomic files are available in the Github repository for this paper (https://github.com/brunetlab/Papsdorf_etal_2021).

\section{Code availability}

The code used to analyze lipidomic data in the current study are available in the Github repository for this paper (https://github.com/brunetlab/Papsdorf_etal_2021).

\section{References}

1 Tosti, V., Bertozzi, B. \& Fontana, L. Health Benefits of the Mediterranean Diet: Metabolic and Molecular Mechanisms. J Gerontol A Biol Sci Med Sci 73, 318-326, doi:10.1093/gerona/glx227 (2018).

2 Mendez, M. A. \& Newman, A. B. Can a Mediterranean Diet Pattern Slow Aging? J Gerontol A Biol Sci Med Sci 73, 315-317, doi:10.1093/gerona/gly003 (2018).

3 Hansen, M., Flatt, T. \& Aguilaniu, H. Reproduction, fat metabolism, and life span: what is the connection? Cell Metab 17, 10-19, doi:10.1016/j.cmet.2012.12.003 (2013).

4 Broughton, S. J. et al. Longer lifespan, altered metabolism, and stress resistance in Drosophila from ablation of cells making insulin-like ligands. Proc Natl Acad Sci US A 102, 3105-3110, doi:10.1073/pnas.0405775102 (2005).

5 Greer, E. L. et al. Members of the H3K4 trimethylation complex regulate lifespan in a germline-dependent manner in C. elegans. Nature 466, 383-387, doi:10.1038/nature09195 (2010).

6 Han, S. et al. Mono-unsaturated fatty acids link H3K4me3 modifiers to C. elegans lifespan. Nature 544, 185-190, doi:10.1038/nature21686 (2017).

$7 \quad$ Folick, A. et al. Aging. Lysosomal signaling molecules regulate longevity in Caenorhabditis elegans. Science 347, 83-86, doi:10.1126/science.1258857 (2015). 
7298 O'Rourke, E. J., Kuballa, P., Xavier, R. \& Ruvkun, G. omega-6 Polyunsaturated fatty

$730 \quad$ acids extend life span through the activation of autophagy. Genes Dev 27, 429-440,

731 doi:10.1101/gad.205294.112 (2013).

7329 Qi, W. et al. The omega-3 fatty acid alpha-linolenic acid extends Caenorhabditis elegans lifespan via NHR-49/PPARalpha and oxidation to oxylipins. Aging Cell 16, 1125-1135, doi:10.1111/acel.12651 (2017).

10 Yuan, Y. et al. Enhanced energy metabolism contributes to the extended life span of calorie-restricted Caenorhabditis elegans. J Biol Chem 287, 31414-31426, doi:10.1074/jbc.M112.377275 (2012).

11 Goudeau, J. et al. Fatty acid desaturation links germ cell loss to longevity through NHR80/HNF4 in C. elegans. PLoS Biol 9, e1000599, doi:10.1371/journal.pbio.1000599 (2011).

12 Choi, L. S., Shi, C., Ashraf, J., Sohrabi, S. \& Murphy, C. T. Oleic Acid Protects Caenorhabditis Mothers From Mating-Induced Death and the Cost of Reproduction. Front Cell Dev Biol 9, 690373, doi:10.3389/fcell.2021.690373 (2021).

13 Cui, W., Liu, D., Gu, W. \& Chu, B. Peroxisome-driven ether-linked phospholipids biosynthesis is essential for ferroptosis. Cell Death Differ, doi:10.1038/s41418-02100769-0 (2021).

14 Zou, Y. et al. Plasticity of ether lipids promotes ferroptosis susceptibility and evasion. Nature 585, 603-608, doi:10.1038/s41586-020-2732-8 (2020).

15 Perez, M. A., Magtanong, L., Dixon, S. J. \& Watts, J. L. Dietary Lipids Induce Ferroptosis in Caenorhabditiselegans and Human Cancer Cells. Dev Cell 54, 447 454.e444, doi:10.1016/j.devcel.2020.06.019 (2020).

16 Cao, J. Y. \& Dixon, S. J. Mechanisms of ferroptosis. Cell Mol Life Sci 73, 2195-2209, doi:10.1007/s00018-016-2194-1 (2016).

17 Aldrovandi, M. \& Conrad, M. Ferroptosis: the Good, the Bad and the Ugly. Cell Res 30, 1061-1062, doi:10.1038/s41422-020-00434-0 (2020).

18 Mutlu, A. S., Duffy, J. \& Wang, M. C. Lipid metabolism and lipid signals in aging and longevity. Dev Cell 56, 1394-1407, doi:10.1016/j.devcel.2021.03.034 (2021).

19 Bustos, V. \& Partridge, L. Good Ol' Fat: Links between Lipid Signaling and Longevity. Trends Biochem Sci 42, 812-823, doi:10.1016/j.tibs.2017.07.001 (2017).

20 Schroeder, E. A. \& Brunet, A. Lipid Profiles and Signals for Long Life. Trends Endocrinol Metab 26, 589-592, doi:10.1016/j.tem.2015.08.007 (2015).

21 Puca, A. A., Chatgilialoglu, C. \& Ferreri, C. Lipid metabolism and diet: possible mechanisms of slow aging. Int J Biochem Cell Biol 40, 324-333, doi:10.1016/j.biocel.2007.04.003 (2008).

22 Gao, Q. \& Goodman, J. M. The lipid droplet-a well-connected organelle. Front Cell Dev Biol 3, 49, doi:10.3389/fcell.2015.00049 (2015).

23 Olzmann, J. A. \& Carvalho, P. Dynamics and functions of lipid droplets. Nat Rev Mol Cell Biol 20, 137-155, doi:10.1038/s41580-018-0085-z (2019).

24 Walther, T. C. \& Farese, R. V., Jr. Lipid droplets and cellular lipid metabolism. Annu Rev Biochem 81, 687-714, doi:10.1146/annurev-biochem-061009-102430 (2012).

25 Marschallinger, J. et al. Lipid-droplet-accumulating microglia represent a dysfunctional and proinflammatory state in the aging brain. Nat Neurosci 23, 194-208, doi:10.1038/s41593-019-0566-1 (2020).

26 Gluchowski, N. L., Becuwe, M., Walther, T. C. \& Farese, R. V., Jr. Lipid droplets and liver disease: from basic biology to clinical implications. Nat Rev Gastroenterol Hepatol 14, 343-355, doi:10.1038/nrgastro.2017.32 (2017). 
808

809

810

811

812

813

814

815

816

817

818

819

820

821

822

27 Liu, L. et al. Glial lipid droplets and ROS induced by mitochondrial defects promote neurodegeneration. Cell 160, 177-190, doi:10.1016/j.cell.2014.12.019 (2015).

28 Geltinger, F. et al. The transfer of specific mitochondrial lipids and proteins to lipid droplets contributes to proteostasis upon stress and aging in the eukaryotic model system Saccharomyces cerevisiae. Geroscience 42, 19-38, doi:10.1007/s 11357-019-00103-0 (2020).

29 Bailey, A. P. et al. Antioxidant Role for Lipid Droplets in a Stem Cell Niche of Drosophila. Cell 163, 340-353, doi:10.1016/j.cell.2015.09.020 (2015).

30 Ugrankar, R. et al. Drosophila Snazarus Regulates a Lipid Droplet Population at Plasma Membrane-Droplet Contacts in Adipocytes. Dev Cell 50, 557-572.e555, doi:10.1016/j.devcel.2019.07.021 (2019).

31 Krahmer, N., Farese, R. V., Jr. \& Walther, T. C. Balancing the fat: lipid droplets and human disease. EMBO Mol Med 5, 973-983, doi:10.1002/emmm.201100671 (2013).

32 Mashek, D. G. Hepatic lipid droplets: A balancing act between energy storage and metabolic dysfunction in NAFLD. Mol Metab, 101115, doi:10.1016/j.molmet.2020.101115 (2020).

33 Ralhan, I., Chang, C. L., Lippincott-Schwartz, J. \& Ioannou, M. S. Lipid droplets in the nervous system. J Cell Biol 220, doi:10.1083/jcb.202102136 (2021).

34 Najt, C. P. et al. Lipid Droplet-Derived Monounsaturated Fatty Acids Traffic via PLIN5 to Allosterically Activate SIRT1. Mol Cell 77, 810-824.e818, doi:10.1016/j.molcel.2019.12.003 (2020).

35 Ramachandran, P. V., Mutlu, A. S. \& Wang, M. C. Label-free biomedical imaging of lipids by stimulated Raman scattering microscopy. Curr Protoc Mol Biol 109, 30.33.3130.33.17, doi:10.1002/0471142727.mb3003s109 (2015).

36 Chen, T., Yavuz, A. \& Wang, M. C. Dissecting lipid droplet biology with coherent Raman scattering microscopy. J Cell Sci 135, doi:10.1242/jcs.252353 (2022).

37 Mutlu, A. S., Chen, T., Deng, D. \& Wang, M. C. Label-Free Imaging of Lipid Storage Dynamics in Caenorhabditis elegans using Stimulated Raman Scattering Microscopy. $J$ Vis Exp, doi:10.3791/61870 (2021).

38 Chen, W. W. et al. Spectroscopic coherent Raman imaging of Caenorhabditis elegans reveals lipid particle diversity. Nat Chem Biol 16, 1087-1095, doi:10.1038/s41589-0200565-2 (2020).

39 Barros, A. G., Liu, J., Lemieux, G. A., Mullaney, B. C. \& Ashrafi, K. Analyses of C. elegans fat metabolic pathways. Methods Cell Biol 107, 383-407, doi:10.1016/b978-0-12394620-1.00013-8 (2012).

$40 \mathrm{Na}, \mathrm{H}$. et al. Identification of lipid droplet structure-like/resident proteins in Caenorhabditis elegans. Biochim Biophys Acta 1853, 2481-2491, doi:10.1016/j.bbamcr.2015.05.020 (2015).

41 Vrablik, T. L., Petyuk, V. A., Larson, E. M., Smith, R. D. \& Watts, J. L. Lipidomic and proteomic analysis of Caenorhabditis elegans lipid droplets and identification of ACS-4 as a lipid droplet-associated protein. Biochim Biophys Acta 1851, 1337-1345, doi:10.1016/j.bbalip.2015.06.004 (2015).

42 Daniele, J. R. et al. UPR(ER) promotes lipophagy independent of chaperones to extend life span. Sci $A d v$ 6, eaaz1441, doi:10.1126/sciadv.aaz1441 (2020).

43 Ganguly, R. \& Pierce, G. N. Trans fat involvement in cardiovascular disease. Mol Nutr Food Res 56, 1090-1096, doi:10.1002/mnfr.201100700 (2012). 
82344 Zhang, P. \& Reue, K. Lipin proteins and glycerolipid metabolism: Roles at the ER

824

825

826

827

828

829

830

831

832

833

834

835

836

837

838

839

840

841

842

843

844

845

846

847

848

849

850

851

852

853

854

855

856

857

858

859

860

861

862

863

864

865

866

867

868 membrane and beyond. Biochim Biophys Acta Biomembr 1859, 1583-1595, doi:10.1016/j.bbamem.2017.04.007 (2017).

45 Donkor, J., Sariahmetoglu, M., Dewald, J., Brindley, D. N. \& Reue, K. Three mammalian lipins act as phosphatidate phosphatases with distinct tissue expression patterns. $J$ Biol Chem 282, 3450-3457, doi:10.1074/jbc.M610745200 (2007).

46 Adeyo, O. et al. The yeast lipin orthologue Pah1p is important for biogenesis of lipid droplets. J Cell Biol 192, 1043-1055, doi:10.1083/jcb.201010111 (2011).

47 Golden, A., Liu, J. \& Cohen-Fix, O. Inactivation of the C. elegans lipin homolog leads to ER disorganization and to defects in the breakdown and reassembly of the nuclear envelope. J Cell Sci 122, 1970-1978, doi:10.1242/jcs.044743 (2009).

48 Wang, H. et al. Lipin-1gamma isoform is a novel lipid droplet-associated protein highly expressed in the brain. FEBS Lett 585, 1979-1984, doi:10.1016/j.febslet.2011.05.035 (2011).

49 Sellers, R. S., Mahmood, S. R., Perumal, G. S., Macaluso, F. P. \& Kurland, I. J. Phenotypic Modulation of Skeletal Muscle Fibers in LPIN1-Deficient Lipodystrophic ( fld) Mice. Vet Pathol 56, 322-331, doi:10.1177/0300985818809126 (2019).

50 Jung, Y. et al. Caenorhabditis elegans Lipin 1 moderates the lifespan-shortening effects of dietary glucose by maintaining $\omega-6$ polyunsaturated fatty acids. Aging Cell 19, e13150, doi:10.1111/acel.13150 (2020).

51 Nettebrock, N. T. \& Bohnert, M. Born this way - Biogenesis of lipid droplets from specialized ER subdomains. Biochim Biophys Acta Mol Cell Biol Lipids 1865, doi:10.1016/j.bbalip.2019.04.008 (2020).

52 Szymanski, K. M. et al. The lipodystrophy protein seipin is found at endoplasmic reticulum lipid droplet junctions and is important for droplet morphology. Proc Natl Acad Sci U S A 104, 20890-20895, doi:10.1073/pnas.0704154104 (2007).

53 Cartwright, B. R. et al. Seipin performs dissectible functions in promoting lipid droplet biogenesis and regulating droplet morphology. Mol Biol Cell 26, 726-739, doi:10.1091/mbc.E14-08-1303 (2015).

54 Wang, H. et al. Seipin is required for converting nascent to mature lipid droplets. Elife 5, doi:10.7554/eLife.16582 (2016).

55 Cao, Z. et al. Dietary fatty acids promote lipid droplet diversity through seipin enrichment in an ER subdomain. Nat Commun 10, 2902, doi:10.1038/s41467-019-108354 (2019).

56 Zimmermann, R. et al. Fat mobilization in adipose tissue is promoted by adipose triglyceride lipase. Science 306, 1383-1386, doi:10.1126/science.1100747 (2004).

57 Yang, L. et al. Neuronal lipolysis participates in PUFA-mediated neural function and neurodegeneration. EMBO Rep 21, e50214, doi:10.15252/embr.202050214 (2020).

58 Sathyanarayan, A., Mashek, M. T. \& Mashek, D. G. ATGL Promotes Autophagy/Lipophagy via SIRT1 to Control Hepatic Lipid Droplet Catabolism. Cell Rep 19, 1-9, doi:10.1016/j.celrep.2017.03.026 (2017).

59 Bazopoulou, D. et al. Developmental ROS individualizes organismal stress resistance and lifespan. Nature 576, 301-305, doi:10.1038/s41586-019-1814-y (2019).

60 Booth, L. N., Maures, T. J., Yeo, R. W., Tantilert, C. \& Brunet, A. Self-sperm induce resistance to the detrimental effects of sexual encounters with males in hermaphroditic nematodes. Elife 8, doi:10.7554/eLife.46418 (2019). 
86961 Rambold, A. S., Cohen, S. \& Lippincott-Schwartz, J. Fatty acid trafficking in starved

870

871

872

873

874

875

876

877

878

879

880

881

882

883

884

885

886

887

888

889

890

891

892

893

894

895

896

897

898

899

900

901

902

903

904

905

906

907

908

909

910

911

912

913

914

915 cells: regulation by lipid droplet lipolysis, autophagy, and mitochondrial fusion dynamics. Dev Cell 32, 678-692, doi:10.1016/j.devcel.2015.01.029 (2015).

62 Nguyen, T. B. et al. DGAT1-Dependent Lipid Droplet Biogenesis Protects Mitochondrial Function during Starvation-Induced Autophagy. Dev Cell 42, 9-21.e25, doi:10.1016/j.devcel.2017.06.003 (2017).

63 Valm, A. M. et al. Applying systems-level spectral imaging and analysis to reveal the organelle interactome. Nature 546, 162-167, doi:10.1038/nature22369 (2017).

64 Cohen, S., Rambold, A. S. \& Lippincott-Schwartz, J. Mitochondrial and Lipid Droplet Dynamics Regulate Intra- and Intercellular Fatty Acid Trafficking. Mol Cell Oncol 5, e1043038, doi:10.1080/23723556.2015.1043038 (2018).

65 Chang, C. L. et al. Spastin tethers lipid droplets to peroxisomes and directs fatty acid trafficking through ESCRT-III. J Cell Biol 218, 2583-2599, doi:10.1083/jcb.201902061 (2019).

66 Weir, H. J. et al. Dietary Restriction and AMPK Increase Lifespan via Mitochondrial Network and Peroxisome Remodeling. Cell Metab 26, 884-896.e885, doi:10.1016/j.cmet.2017.09.024 (2017).

67 Huang, K. et al. Impaired peroxisomal import in Drosophila oenocytes causes cardiac dysfunction by inducing upd3 as a peroxikine. Nat Commun 11, 2943, doi:10.1038/s41467-020-16781-w (2020).

68 Zhang, S. O. et al. Genetic and dietary regulation of lipid droplet expansion in Caenorhabditis elegans. Proc Natl Acad Sci U S A 107, 4640-4645, doi:10.1073/pnas.0912308107 (2010).

69 Kong, J. et al. Spatiotemporal contact between peroxisomes and lipid droplets regulates fasting-induced lipolysis via PEX5. Nat Commun 11, 578, doi:10.1038/s41467-01914176-0 (2020).

70 Narayan, V. et al. Deep Proteome Analysis Identifies Age-Related Processes in C. elegans. Cell Syst 3, 144-159, doi:10.1016/j.cels.2016.06.011 (2016).

71 Bersuker, K. et al. A Proximity Labeling Strategy Provides Insights into the Composition and Dynamics of Lipid Droplet Proteomes. Dev Cell 44, 97-112.e117, doi:10.1016/j.devcel.2017.11.020 (2018).

72 Watts, J. L. \& Ristow, M. Lipid and Carbohydrate Metabolism in Caenorhabditis elegans. Genetics 207, 413-446, doi:10.1534/genetics.117.300106 (2017).

73 Boström, P. et al. SNARE proteins mediate fusion between cytosolic lipid droplets and are implicated in insulin sensitivity. Nat Cell Biol 9, 1286-1293, doi:10.1038/ncb1648 (2007).

74 Chung, J. et al. LDAF1 and Seipin Form a Lipid Droplet Assembly Complex. Dev Cell 51, 551-563.e557, doi:10.1016/j.devcel.2019.10.006 (2019).

75 Walther, T. C., Chung, J. \& Farese, R. V., Jr. Lipid Droplet Biogenesis. Annu Rev Cell Dev Biol 33, 491-510, doi:10.1146/annurev-cellbio-100616-060608 (2017).

76 Coppa, A. et al. The peroxisomal fatty acid transporter ABCD1/PMP-4 is required in the C. elegans hypodermis for axonal maintenance: A worm model for adrenoleukodystrophy. Free Radic Biol Med 152, 797-809, doi:10.1016/j.freeradbiomed.2020.01.177 (2020).

77 Thieringer, H., Moellers, B., Dodt, G., Kunau, W. H. \& Driscoll, M. Modeling human peroxisome biogenesis disorders in the nematode Caenorhabditis elegans. J Cell Sci 116, 1797-1804, doi:10.1242/jcs.00380 (2003). 
91678 Choudhary, V., Ojha, N., Golden, A. \& Prinz, W. A. A conserved family of proteins

917

918

919

920

921

922

923

924

925

926

927

928

929

930

931

932

933

934

935

936

937

938

939

940

941

942

943

944

945

946

947

948

949

950

951

952

953

954

955

956

957

958

959

960

961 facilitates nascent lipid droplet budding from the ER. J Cell Biol 211, 261-271, doi:10.1083/jcb.201505067 (2015).

79 Shi, X. et al. A Caenorhabditis elegans model for ether lipid biosynthesis and function. $J$ Lipid Res 57, 265-275, doi:10.1194/jlr.M064808 (2016).

80 Nguyen, T. B. \& Olzmann, J. A. Getting a handle on lipid droplets: Insights into ER-lipid droplet tethering. J Cell Biol 218, 1089-1091, doi:10.1083/jcb.201902160 (2019).

81 Joshi, A. S. \& Cohen, S. Lipid Droplet and Peroxisome Biogenesis: Do They Go Handin-Hand? Front Cell Dev Biol 7, 92, doi:10.3389/fcell.2019.00092 (2019).

82 Joshi, A. S. et al. Lipid droplet and peroxisome biogenesis occur at the same ER subdomains. Nat Commun 9, 2940, doi:10.1038/s41467-018-05277-3 (2018).

83 Butcher, R. A. et al. Biosynthesis of the Caenorhabditis elegans dauer pheromone. Proc Natl Acad Sci U S A 106, 1875-1879, doi:10.1073/pnas.0810338106 (2009).

84 Artyukhin, A. B. et al. Metabolomic "Dark Matter" Dependent on Peroxisomal $\beta$ Oxidation in Caenorhabditis elegans. J Am Chem Soc 140, 2841-2852, doi:10.1021/jacs.7b11811 (2018).

85 Bouagnon, A. D. et al. Intestinal peroxisomal fatty acid $\beta$-oxidation regulates neural serotonin signaling through a feedback mechanism. PLoS Biol 17, e3000242, doi:10.1371/journal.pbio.3000242 (2019).

86 Xie, M. \& Roy, R. Increased levels of hydrogen peroxide induce a HIF-1-dependent modification of lipid metabolism in AMPK compromised C. elegans dauer larvae. Cell Metab 16, 322-335, doi:10.1016/j.cmet.2012.07.016 (2012).

87 Lee, H. et al. Energy-stress-mediated AMPK activation inhibits ferroptosis. Nat Cell Biol 22, 225-234, doi:10.1038/s41556-020-0461-8 (2020).

88 Jenkins, N. L. et al. Changes in ferrous iron and glutathione promote ferroptosis and frailty in aging Caenorhabditis elegans. Elife 9, doi:10.7554/eLife.56580 (2020).

89 Jiang, X., Stockwell, B. R. \& Conrad, M. Ferroptosis: mechanisms, biology and role in disease. Nat Rev Mol Cell Biol 22, 266-282, doi:10.1038/s41580-020-00324-8 (2021).

90 Stockwell, B. R., Jiang, X. \& Gu, W. Emerging Mechanisms and Disease Relevance of Ferroptosis. Trends Cell Biol 30, 478-490, doi:10.1016/j.tcb.2020.02.009 (2020).

91 Forcina, G. C. \& Dixon, S. J. GPX4 at the Crossroads of Lipid Homeostasis and Ferroptosis. Proteomics 19, e1800311, doi:10.1002/pmic.201800311 (2019).

92 Bosch, M. et al. Mammalian lipid droplets are innate immune hubs integrating cell metabolism and host defense. Science 370, doi:10.1126/science.aay8085 (2020).

93 Geltinger, F. et al. Friend or Foe: Lipid Droplets as Organelles for Protein and Lipid Storage in Cellular Stress Response, Aging and Disease. Molecules 25, doi:10.3390/molecules25215053 (2020).

94 Ioannou, M. S. et al. Neuron-Astrocyte Metabolic Coupling Protects against ActivityInduced Fatty Acid Toxicity. Cell 177, 1522-1535.e1514, doi:10.1016/j.cell.2019.04.001 (2019).

95 Shimabukuro, M. K. et al. Lipid-laden cells differentially distributed in the aging brain are functionally active and correspond to distinct phenotypes. Sci Rep 6, 23795, doi:10.1038/srep23795 (2016).

96 Mosquera, J. V., Bacher, M. C. \& Priess, J. R. Nuclear lipid droplets and nuclear damage in Caenorhabditis elegans. PLoS Genet 17, e1009602, doi:10.1371/journal.pgen.1009602 (2021). 
96297 Lapierre, L. R., Gelino, S., Meléndez, A. \& Hansen, M. Autophagy and lipid metabolism

963

964

965

966

967

968

969

970

971

972

973

974

975

976

977

978

979

980

981

982

983

984

985

986

987

988

989

990

991

992

993

994

995

996

997

998

999

1000

1001

1002

1003

1004

1005

1006

1007

1008

1009 coordinately modulate life span in germline-less C. elegans. Curr Biol 21, 1507-1514, doi:10.1016/j.cub.2011.07.042 (2011).

98 Seah, N. E. et al. Autophagy-mediated longevity is modulated by lipoprotein biogenesis. Autophagy 12, 261-272, doi:10.1080/15548627.2015.1127464 (2016).

99 Lee, G. et al. Post-transcriptional Regulation of De Novo Lipogenesis by mTORC1S6K1-SRPK2 Signaling. Cell 171, 1545-1558.e1518, doi:10.1016/j.cell.2017.10.037 (2017).

100 Steinbaugh, M. J. et al. Lipid-mediated regulation of SKN-1/Nrf in response to germ cell absence. Elife 4, doi:10.7554/eLife.07836 (2015).

101 Lynn, D. A. et al. Omega-3 and -6 fatty acids allocate somatic and germline lipids to ensure fitness during nutrient and oxidative stress in Caenorhabditis elegans. Proc Natl Acad Sci U S A 112, 15378-15383, doi:10.1073/pnas.1514012112 (2015).

102 Lapierre, L. R., Meléndez, A. \& Hansen, M. Autophagy links lipid metabolism to longevity in C. elegans. Autophagy 8, 144-146, doi:10.4161/auto.8.1.18722 (2012).

$103 \mathrm{Kim}, \mathrm{H}$. E. et al. Lipid Biosynthesis Coordinates a Mitochondrial-to-Cytosolic Stress Response. Cell 166, 1539-1552.e1516, doi:10.1016/j.cell.2016.08.027 (2016).

104 Higuchi-Sanabria, R. et al. Divergent Nodes of Non-autonomous UPR(ER) Signaling through Serotonergic and Dopaminergic Neurons. Cell Rep 33, 108489, doi:10.1016/j.celrep.2020.108489 (2020).

105 Goh, G. Y. S. et al. NHR-49/HNF4 integrates regulation of fatty acid metabolism with a protective transcriptional response to oxidative stress and fasting. Aging Cell 17, e12743, doi:10.1111/acel.12743 (2018).

106 Imanikia, S., Sheng, M., Castro, C., Griffin, J. L. \& Taylor, R. C. XBP-1 Remodels Lipid Metabolism to Extend Longevity. Cell Rep 28, 581-589.e584, doi:10.1016/j.celrep.2019.06.057 (2019).

107 Webster, C. M. et al. Genome-wide RNAi Screen for Fat Regulatory Genes in C. elegans Identifies a Proteostasis-AMPK Axis Critical for Starvation Survival. Cell Rep 20, 627640, doi:10.1016/j.celrep.2017.06.068 (2017).

108 Heimbucher, T., Hog, J., Gupta, P. \& Murphy, C. T. PQM-1 controls hypoxic survival via regulation of lipid metabolism. Nat Commun 11, 4627, doi:10.1038/s41467-02018369-w (2020).

109 Zhang, P., Judy, M., Lee, S. J. \& Kenyon, C. Direct and indirect gene regulation by a lifeextending FOXO protein in C. elegans: roles for GATA factors and lipid gene regulators. Cell Metab 17, 85-100, doi:10.1016/j.cmet.2012.12.013 (2013).

110 Littlejohn, N. K., Seban, N., Liu, C. C. \& Srinivasan, S. A feedback loop governs the relationship between lipid metabolism and longevity. Elife 9, doi:10.7554/eLife.58815 (2020).

111 Wang, M. C., O'Rourke, E. J. \& Ruvkun, G. Fat metabolism links germline stem cells and longevity in C. elegans. Science 322, 957-960, doi:10.1126/science.1162011 (2008).

112 Chen, A. L. et al. Pharmacological convergence reveals a lipid pathway that regulates C. elegans lifespan. Nat Chem Biol 15, 453-462, doi:10.1038/s41589-019-0243-4 (2019).

113 Lee, D. et al. MDT-15/MED15 permits longevity at low temperature via enhancing lipidostasis and proteostasis. PLoS Biol 17, e3000415, doi:10.1371/journal.pbio.3000415 (2019).

114 Soukas, A. A., Kane, E. A., Carr, C. E., Melo, J. A. \& Ruvkun, G. Rictor/TORC2 regulates fat metabolism, feeding, growth, and life span in Caenorhabditis elegans. Genes Dev 23, 496-511, doi:10.1101/gad.1775409 (2009). 
1010

1011

1012

1013

1014

1015

1016

1017

1018

1019

1020

1021

1022

1023

1024

1025

1026

1027

1028

1029

1030

1031

1032

1033

1034

1035

1036

1037

1038

1039

1040

1041

1042

1043

1044

1045

1046

1047

1048

1049

1050

1051

1052

1053

1054

1055

1056

1057

115 Liao, C. Y. et al. Fat maintenance is a predictor of the murine lifespan response to dietary restriction. Aging Cell 10, 629-639, doi:10.1111/j.1474-9726.2011.00702.x (2011).

116 Zheng, H., Echave, P., Mehta, N. \& Myrskylä, M. Life-long body mass index trajectories and mortality in two generations. Ann Epidemiol 56, 18-25, doi:10.1016/j.annepidem.2021.01.003 (2021).

117 Magtanong, L. et al. Exogenous Monounsaturated Fatty Acids Promote a FerroptosisResistant Cell State. Cell Chem Biol 26, 420-432.e429, doi:10.1016/j.chembiol.2018.11.016 (2019).

118 Silva-García, C. G. et al. Single-Copy Knock-In Loci for Defined Gene Expression in Caenorhabditis elegans. G3 (Bethesda) 9, 2195-2198, doi:10.1534/g3.119.400314 (2019).

119 Yu, Y., Mutlu, A. S., Liu, H. \& Wang, M. C. High-throughput screens using photohighlighting discover BMP signaling in mitochondrial lipid oxidation. Nat Commun $\mathbf{8}$, 865, doi:10.1038/s41467-017-00944-3 (2017).

120 Schindelin, J. et al. Fiji: an open-source platform for biological-image analysis. Nat Methods 9, 676-682, doi:10.1038/nmeth.2019 (2012).

121 Rizk, A. et al. Segmentation and quantification of subcellular structures in fluorescence microscopy images using Squassh. Nat Protoc 9, 586-596, doi:10.1038/nprot.2014.037 (2014).

122 Chen, E. Y. et al. Enrichr: interactive and collaborative HTML5 gene list enrichment analysis tool. BMC Bioinformatics 14, 128, doi:10.1186/1471-2105-14-128 (2013).

123 Kuleshov, M. V. et al. Enrichr: a comprehensive gene set enrichment analysis web server 2016 update. Nucleic Acids Res 44, W90-97, doi:10.1093/nar/gkw377 (2016).

124 Kuleshov, M. V. et al. modEnrichr: a suite of gene set enrichment analysis tools for model organisms. Nucleic Acids Res 47, W183-w190, doi:10.1093/nar/gkz347 (2019).

125 Matyash, V., Liebisch, G., Kurzchalia, T. V., Shevchenko, A. \& Schwudke, D. Lipid extraction by methyl-tert-butyl ether for high-throughput lipidomics. J Lipid Res $\mathbf{4 9}$, 1137-1146, doi:10.1194/jlr.D700041-JLR200 (2008).

126 Taguchi, R. \& Ishikawa, M. Precise and global identification of phospholipid molecular species by an Orbitrap mass spectrometer and automated search engine Lipid Search. $J$ Chromatogr A 1217, 4229-4239, doi:10.1016/j.chroma.2010.04.034 (2010).

127 Rackles, E. et al. Reduced peroxisomal import triggers peroxisomal retrograde signaling. Cell Rep 34, 108653, doi:10.1016/j.celrep.2020.108653 (2021).

128 Chen, C. et al. Pex11 a deficiency causes dyslipidaemia and obesity in mice. J Cell Mol Med 23, 2020-2031, doi:10.1111/jcmm.14108 (2019).

129 Abe, Y., Honsho, M., Nakanishi, H., Taguchi, R. \& Fujiki, Y. Very-long-chain polyunsaturated fatty acids accumulate in phosphatidylcholine of fibroblasts from patients with Zellweger syndrome and acyl-CoA oxidase1 deficiency. Biochim Biophys Acta 1841, 610-619, doi:10.1016/j.bbalip.2014.01.001 (2014).

130 Perez, M. A., Magtanong, L., Dixon, S. J. \& Watts, J. L. Dietary Induction and Modulation of Ferroptosis in $<\mathrm{em}>$ Caenorhabditis elegans $</ \mathrm{em}>$. bioRxiv, 772079, doi:10.1101/772079 (2019).

131 Neve, I. A. A. et al. Escherichia coli Metabolite Profiling Leads to the Development of an RNA Interference Strain for Caenorhabditis elegans. G3 (Bethesda) 10, 189-198, doi:10.1534/g3.119.400741 (2020).

132 Kalinowski, D. S. \& Richardson, D. R. The evolution of iron chelators for the treatment of iron overload disease and cancer. Pharmacol Rev 57, 547-583, doi:10.1124/pr.57.4.2 (2005). 
a

Longevity interventions

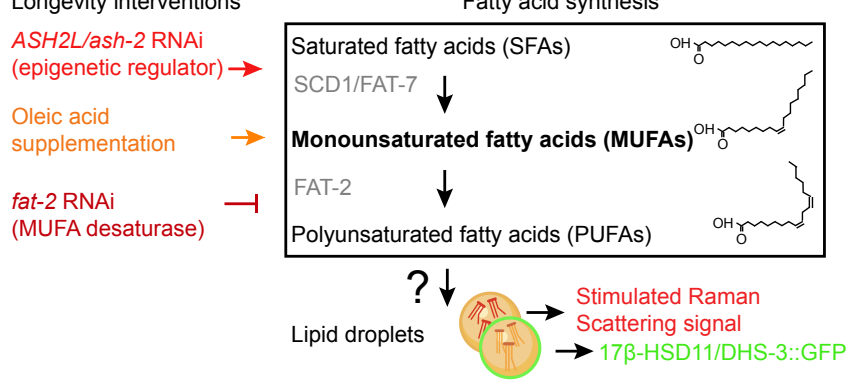

d

Lipid droplet GFP

(DHS-3::GFP)
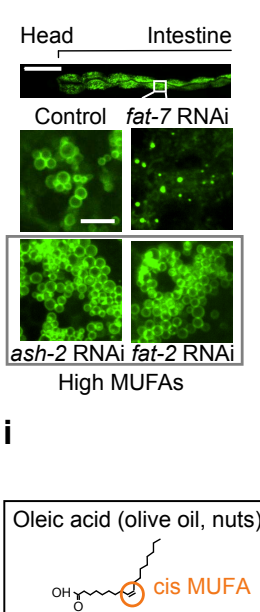

Elaidic acid (margarine, dairy)

trans MUFA e

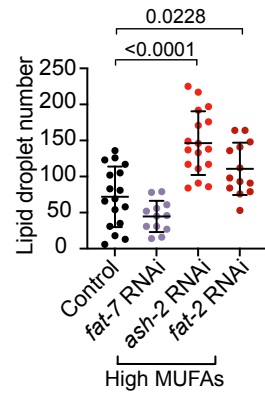

j

Lipid droplet GFP (DHS-3::GFP)

Control Contro (example 1)(example 2)

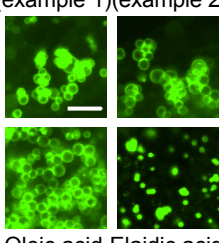

b

Stimulated Raman

Scattering (total lipids)

Head Intestine

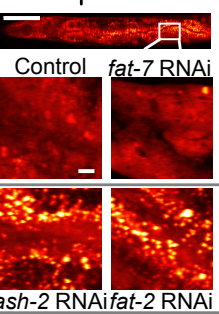

High MUFAs

g

Hypodermal

lipid droplet-mCherry

(PLIN-1::mCherry)

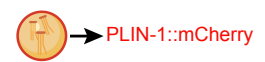

Control Control (example 1)(example 2)

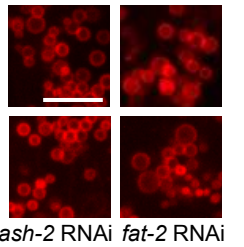



h

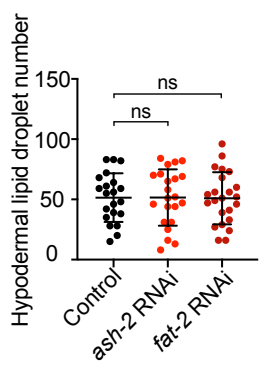

k
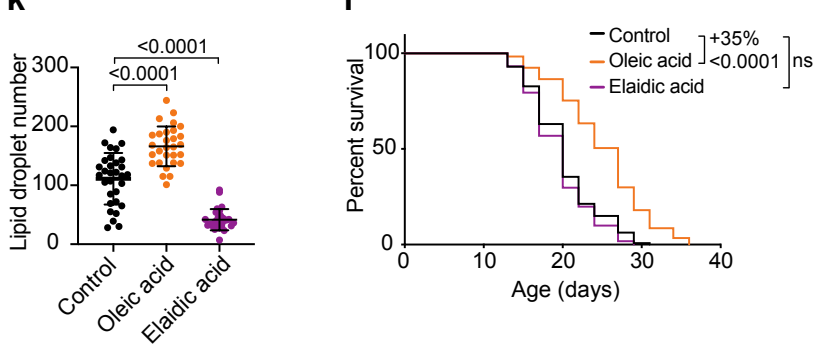

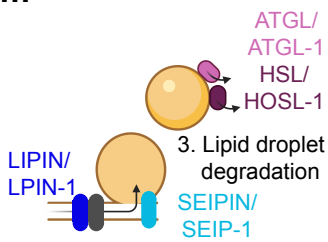

$\begin{array}{ll}\text { 1. Triglyceride } 2 \text {. Lipid droplet } & \end{array}$ synthesis

n

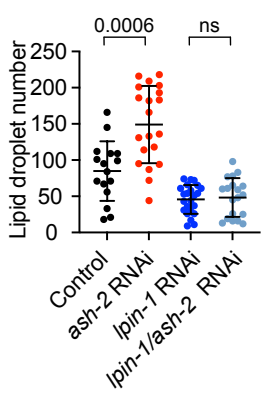

q

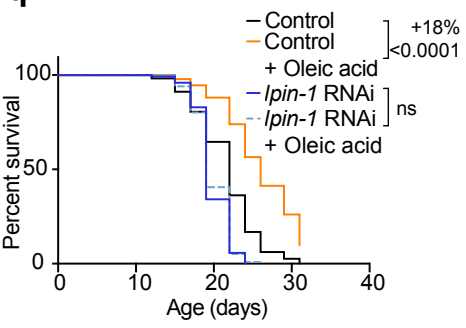

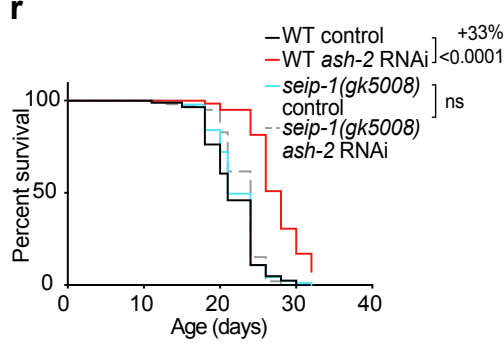

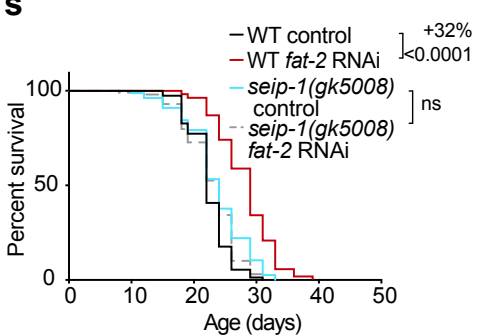


1060

1061

1062

1063

1064

1065

1066

1067

1068

1069

1070

1071

1072

1073

1074

1075

1076

1077

1078

1079

1080

1081

1082

1083

1084

1085

1086

1087

1088

1089

1090

1091

Figure 1. Monounsaturated fatty acids lead to an increase in lipid droplet number and this is necessary for lifespan extension

a, Fatty acid synthesis pathway and lipid droplets in C. elegans and genetic or dietary interventions that lead to accumulation of endogenous monounsaturated fatty acids (MUFAs) ${ }^{6,10}$. The name of the mammalian proteins is also indicated.

b-c, Lipid droplet number and intensity, measured by Stimulated Raman Scattering (SRS) microscopy, in MUFA-enriched worms. b, Upper panel: SRS image of the anterior part of one worm (head and intestine). Scale bar $=100 \mu \mathrm{m}$. Lower panels: Zoomed-in SRS images of worms treated with control (empty vector) RNAi, fat-7 RNAi (MUFA-depleted), and ash-2 or fat-2 RNAi (MUFA-enriched). Scale bar $=5 \mu \mathrm{m}$. c, Quantification of lipid droplet number, as assessed by SRS, of worms treated as in b. $n \geq 18$ worms for each condition. Representative of two independent experiments (see Source Data Figure 1 for all experiments and statistics). Data are mean \pm s.d. Each dot represents the lipid droplet number in a $26 \times 26 \mu \mathrm{m}^{2}$ area in the intestine of an individual worm. $P$-values: two-tailed Mann-Whitney test. Lipid droplet intensity, as assessed by SRS, is quantified in Extended Data Fig. 1d.

d-e, Intestinal lipid droplet number, measured using the transgenic line strain expressing a fusion between the lipid droplet protein DHS-3 and GFP driven by the endogenous $d h s-3$ promoter (intestinal expression) ( $d h s-3 p:: d h s-3:: G F P)$, in MUFA-enriched worms. d, Upper panel: Fluorescent image of the anterior part of one worm (Head and intestine). Scale bar $=100 \mu \mathrm{m}$. Lower panels: Zoomed-in fluorescent images of the mid-intestine area of worms treated with control (empty vector) RNAi, fat-7 RNAi (MUFA-depleted), or ash-2 or fat-2 RNAi (MUFAenriched). Scale bar $=5 \mu \mathrm{m}$. e, Quantification of lipid droplet number in worms treated as in d. $n$ $\geq 12$ worms for each condition. Representative of two independent experiments (see Source Data Figure 1 for all experiments and statistics). Data are mean \pm s.d. Each dot represents the lipid droplet number in a $26 \times 26 \mu \mathrm{m}^{2}$ area in the intestine of an individual worm. $P$-values: two-tailed Mann-Whitney test.

f, Quantification of lipid droplet number in males treated with control (empty vector) RNAi or ash-2 RNAi (MUFA-enriched). $n \geq 22$ worms for each condition. Representative of two independent experiments (see Source Data Figure 1 for all experiments and statistics). Data and $P$-values as in e.

g-h, Hypodermal lipid droplet number in MUFA-enriched worms, measured using the transgenic line strain expressing a fusion between PLIN-1 and mCherry driven by the endogenous plin-1 
1092

1093

1094

1095

1096

1097

1098

1099

1100

1101

1102

1103

1104

1105

1106

1107

1108

1109

1110

1111

1112

1113

1114

1115

1116

1117

1118

1119

1120

1121

1122

1123

promoter (hypodermal expression) (plin-1::plin-1::mCherry). g, Zoomed-in fluorescent image of the hypodermis of worms treated with control (empty vector) RNAi, or ash-2 or fat-2 RNAi (MUFA-enriched). Scale bar $=5 \mu \mathrm{m}$. h, Quantification of lipid droplet number in worms treated as in g. $n \geq 21$ worms for each condition. Representative of two independent experiments (see Source Data Figure 1 for all experiments and statistics). Data are mean \pm s.d. Each dot represents the lipid droplet number in a $15 \times 15 \mu \mathrm{m}^{2}$ area of an individual worm. $P$-values: two-tailed Mann-Whitney test.

i, Chemical structure of the cis MUFA oleic acid and the trans MUFA elaidic acid.

$\mathbf{j}-\mathbf{k}$, Cis MUFA (oleic acid), but not trans MUFA (elaidic acid), increases lipid droplet numbers. Intestinal lipid droplets measured by fluorescence in the $d h s-3 p: \because d h s-3: \because G F P$ transgenic line upon sterically different dietary MUFAs. j, Zoomed-in fluorescent images of the mid-intestine upon dietary oleic acid (cis fatty acid) or elaidic acid (trans fatty acid). Scale bar $=5 \mu \mathrm{m} . \mathbf{k}$, Quantification of lipid droplet number in worms treated as in j. $n \geq 28$ worms for each condition. Representative of two independent experiments (see Source Data Figure 1 for all experiments and statistics). Data and $P$-values as in e.

1, Cis MUFA (oleic acid), but not trans MUFA (elaidic acid), extends lifespan. Kaplan Meier survival curve of worms treated as in $\mathrm{j} . \mathrm{n} \geq 128$ worms for each condition. Representative of two independent experiments (see Extended Data Table 1 for all lifespan experiments and statistics). Percentages of median lifespan extension and $P$-values are indicated on the right. $P$-values: $\log$ rank Mantel-Cox test.

$\mathbf{m}$, Conserved proteins involved in lipid droplet synthesis and degradation. The mammalian name is indicated first. LIPIN/LPIN-1 is part of the pathway that synthesizes the triglycerides packed in lipid droplets. SEIPIN/SEIP-1 assists the budding of mature lipid droplets from the endoplasmic reticulum. The lipases ATGL/ATGL-1 and HSL/HOSL-1 hydrolyze glycerolipids from lipid droplets.

n-o, Lpin-1 is required for lipid droplet increase upon MUFA increase. Intestinal lipid droplet number, measured by fluorescence in the $d h s-3 p:: d h s-3:: G F P$ transgenic line, in MUFAenriched and lipid droplet depleted worms. n, Quantification of lipid droplet number in worms treated with control (empty vector), ash-2, lpin-1, or ash-2/lpin-1 RNAi (see Extended Data Fig. $1 \mathrm{k}$ for efficiency of double knock-down). $n \geq 16$ worms for each condition. Representative of two independent experiments (see Source Data Figure 1 for all experiments and statistics). Data are mean \pm s.d. Each dot represents the lipid droplet number in a $26 \times 26 \mu \mathrm{m}^{2}$ area in the 
1124

1125

1126

1127

1128

1129

1130

1131

1132

1133

1134

1135

1136

1137

1138

1139

1140

1141

1142

1143

1144

1145

1146

1147

1148

1149

1150

1151

1152

1153

1154

intestine of an individual worm. $P$-values: two-tailed Mann-Whitney test. o, Lpin- 1 is required for lipid droplet increase upon oleic acid supplementation. Quantification of lipid droplet number in worms treated with control (empty vector) or lpin-1 RNAi upon dietary oleic acid. $n \geq 29$ worms for each condition. Representative of two independent experiments (see Source Data Figure 1 for all experiments and statistics). Data and $P$-values as in $\mathrm{n}$.

p, lpin-1 is necessary for longevity upon MUFA enrichment by ash-2 depletion. Kaplan Meier survival curve of worms treated with control (empty vector), ash-2, lpin-1, or ash-2/lpin-1 RNAi. $n \geq 96$ for each condition. Representative of two independent experiments (see Extended Data Table 1 for all lifespan experiments and statistics). Percentages of median lifespan extension and $P$-values are indicated on the right. $P$-values: log-rank Mantel-Cox test. ash-2 and lpin-1 RNAi significantly interact with each other using the Cox proportional hazard test $(P$ value $=0.0142$ ).

q, lpin-1 is required for longevity upon oleic acid supplementation. Kaplan Meier survival curve of worms treated with control (empty vector) or lpin-1 RNAi upon dietary oleic acid.

Representative of two independent experiments (see Extended Data Table 1 for all lifespan experiments and statistics). $n \geq 105$ worms for each condition. Percentages of median lifespan extension and $P$-values are indicated on the right. $P$-values: log-rank Mantel-Cox test. Oleic acid and lpin-1 RNAi significantly interact with each other using the Cox proportional hazard test $(P$ value $=2.53 \mathrm{e}-07)$.

r, seip- 1 is required for MUFA-mediated longevity upon ash-2 depletion. Kaplan Meier survival curve of wildtype and seip-1(gk5008) mutant worms treated with control (empty vector) or ash2 RNAi. $\mathrm{n} \geq 64$ for each condition. Representative of three independent experiments (see Extended Data Table 1 for all lifespan experiments and statistics). Percentage of median lifespan extension and $P$-values are indicated on the right. $P$-values: log-rank Mantel-Cox test. ash-2

RNAi and seip-1 mutation significantly interact with each other using the Cox proportional hazard test $(P$-value $=5.36 \mathrm{e}-10)$.

s, seip- 1 is required for MUFA-mediated longevity upon fat-2 depletion. Kaplan Meier survival curve of wildtype and seip-1(gk5008) mutant worms treated with control (empty vector) or fat-2 RNAi. $\mathrm{n} \geq 90$ for each condition. Representative of two independent experiments (see Extended Data Table 1 for all lifespan experiments and statistics). Percentage of median lifespan extension and $P$-values are indicated on the right. $P$-values: log-rank Mantel-Cox test. fat-2 RNAi and seip- 
11551 mutation significantly interact with each other using the Cox proportional hazard test $(P$ 1156 value $=9.07 \mathrm{e}-12$ ).

1157 All experiment data and statistics are shown in Source Data Figure 1. All lifespan data and 1158 statistics are shown in Extended Data Table 1.

1159

1160 

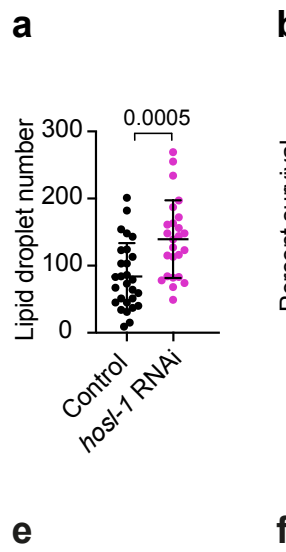

Sorted young adult

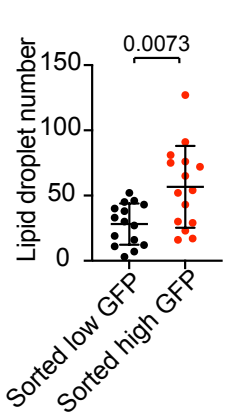

b

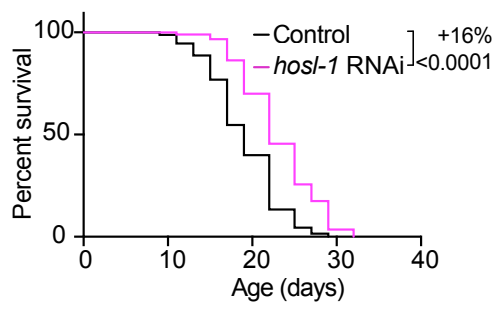

f

Sorted young adult

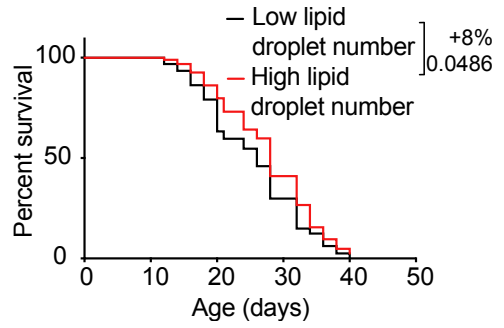

C

Lipid droplet GFP
(DHS-3::GFP)

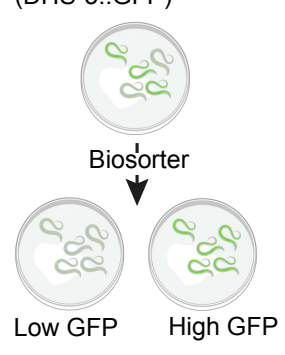

d

Sorted young adult

Low GFP

(low lipid droplet number) (example 1) (example 2)



High GFP (high lipid droplet number) (example 1) (example 2)

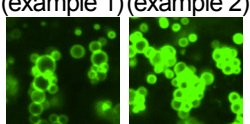

Figure 2. Increased lipid droplets are beneficial for longevity and predictive of a long life

a, hosl-1 knockdown leads to increased lipid droplet number. Intestinal lipid droplet number, measured by fluorescence in the $d h s-3 p:: d h s-3:: G F P$ transgenic line, in worms with decreased lipid droplet degradation. Quantification of lipid droplet number in worms treated with control (empty vector) and hosl-1 RNAi. $n \geq 28$ worms for each condition. Representative of two independent experiments (see Source Data Figure 2 for all experiment data and statistics). Data are mean \pm s.d. Each dot represents the lipid droplet number in a $26 \times 26 \mu \mathrm{m}^{2}$ area in the intestine of an individual worm. $P$-values: two-tailed Mann-Whitney test. with control (empty vector) or hosl-1 RNAi. $\mathrm{n} \geq 94$ for each condition. Representative of three independent experiments (see Extended Data Table 1 for all lifespan experiments and statistics). Percentages of median lifespan extension and $P$-values are indicated on the right. $P$-values: $\log$ rank Mantel-Cox test. particle BioSorter. 
1179

1180

1181

1182

1183

1184

1185

1186

1187

1188

1189

1190

1191

1192

1193

1194

1195

1196

1197

1198

1199

1200

1201

1202

1203

1204

1205

1206

1207

1208

1209

1210

d-e, When sorted at young adult age, high fluorescent DHS-3::GFP worms have a higher intestinal lipid droplet number than low fluorescent worms. Lipid droplet number was assessed after BioSorting a synchronized population of young adult worms (adult day 1) of the $d h s$ $3 p:: d h s-3:: G F P$ transgenic line. d, Zoomed-in fluorescent images of the mid-intestine area. Scale bar $=5 \mu \mathrm{m}$. e, Quantification of lipid droplet number in worms sorted as in d. $n \geq 15$ worms for each condition. Representative of two independent experiments (see Source Data Figure 2 for all experiment data and statistics). Data and $P$-values as in a.

f, Worms sorted at young adult age with high lipid droplet number live longer than worms with a low lipid droplet number. Kaplan Meier survival curve of age-synchronized wildtype worms BioSorted according to their lipid droplet intensity (a proxy for increased lipid droplet number, see e). Representative of two independent experiments (see Extended Data Table 1 for all lifespan experiments and statistics). $n \geq 117$ worms for each condition. The percentage of median lifespan extension are indicated on the right. $P$-values: log-rank Mantel-Cox test.

g, When sorted at middle age, high fluorescent DHS-3::GFP worms have a higher intestinal lipid droplet number than low fluorescent worms. Lipid droplet number was assessed after manual sorting of a synchronized population of middle-aged adult worms (adult day 6) of the $d h s$ $3 p:: d h s-3: \because G F P$ transgenic line. Because the BioSorter requires a high number of worms as an input and because the retrieval of sufficiently high enough worm numbers at old age can be very labor intensive, manual sorting was performed instead. $n \geq 30$ worms for each condition. Representative of two independent experiments (see Source Data Figure 2 for all experiment data and statistics). Data and $P$-values as in a. Zoomed-in images shown in Extended Data Fig $2 \mathrm{~d}$.

h, Worms sorted at middle age with high lipid droplet number live longer than worms with a low lipid droplet number. Kaplan Meier survival curve of age-synchronized wildtype worms manually sorted according to their lipid droplet intensity (a proxy for increased lipid droplet number, see g). $n \geq 195$ worms for each condition. Representative of three independent experiments (see Extended Data Table 1 for all lifespan experiments and statistics). Percentages of median lifespan extension and $P$-values are indicated on the right. $P$-values: log-rank MantelCox test.

All experiment data and statistics are in Source Data Figure 2. All lifespan data and statistics are in Extended Data Table 1. 


\section{Figure 3}

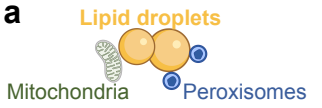

b Shared upregulated GO terms



GFP targeted

to peroxisomes

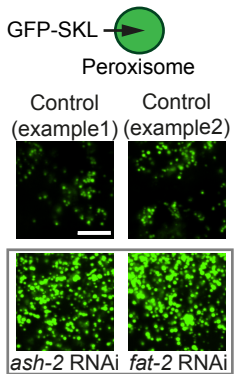

High MUFAs d

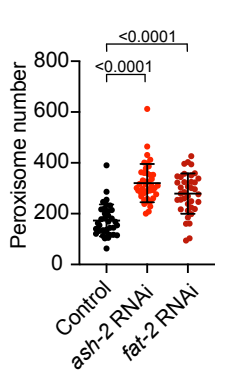

High MUFAs (ash-2 RNAi) and peroxisome disruption (prx-5 RNAi)

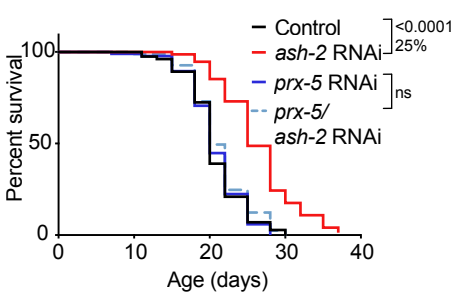

f

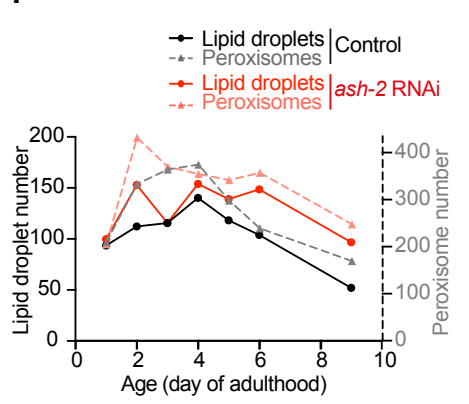

Lipid droplet number

- significant increase

- significant decrease

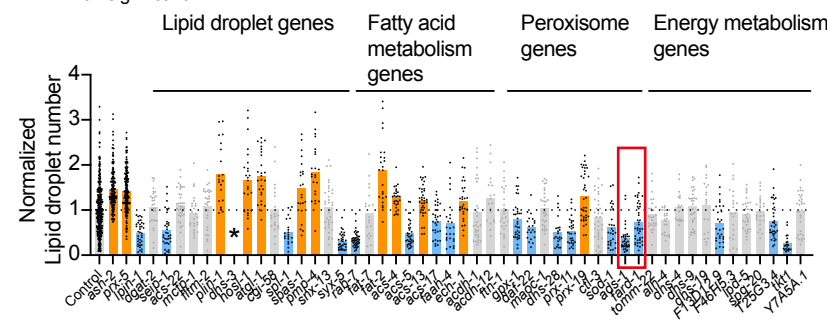

k

Peroxisome number

- significant increase

non significant



m

High MUFAs (ash-2 RNAi) and Ether lipid disruption (ads-1 RNAi)

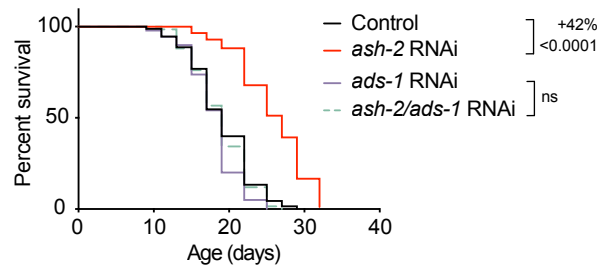
peroxisomes, and metabolism g
Targeted screen

Knock-down of 50 genes related to lipid droplets,

Lipid droplets

(DHS-3::GFP)

Peroxisomes

(RFP-SKL)

$\overrightarrow{\text { igh MUFAs }}$

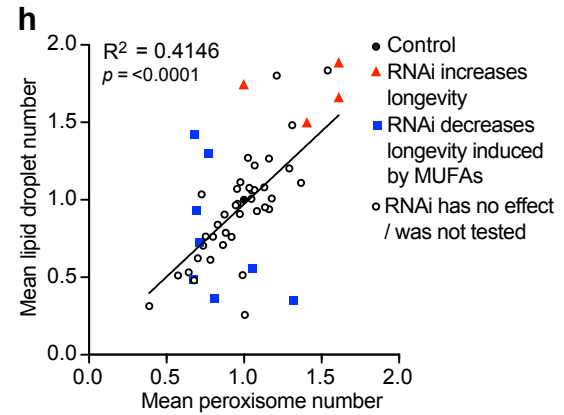

j

RNAi regulating lipid droplet numbers

RNAi decreases lipid droplet number

RNAi increases lipid droplet number

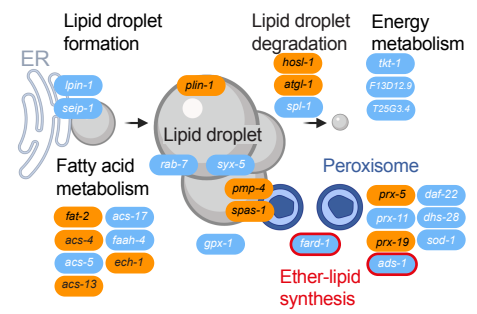

I

RNAi regulating peroxisome numbers

RNAi decreases peroxisome number

RNAi increases peroxisome number

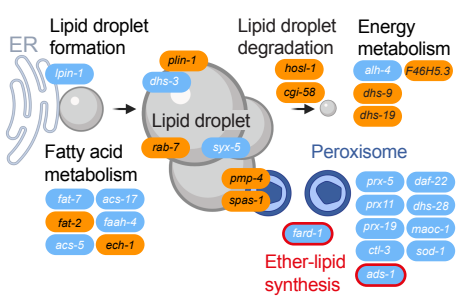

1212

High lipid droplets (hosl-1 RNAi) and Ether lipid disruption (ads-1 RNAi)

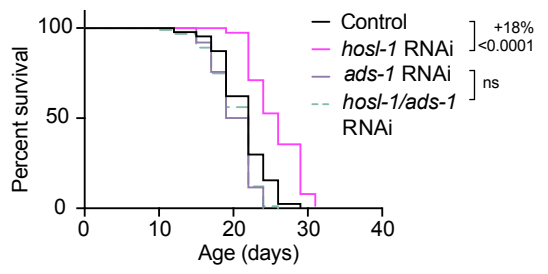


1213 Figure 3. Functional peroxisomes and ether lipid synthesis are required for lipid droplets to extend lifespan in response to MUFAs

1215 a, Organelles such as mitochondria and peroxisomes are in close contact with lipid droplets to 1216 regulate lipid metabolism.

1217 b, Re-analysis of transcriptomic datasets of worms with or without MUFA accumulation ${ }^{5,6}$

1218 Shared upregulated GO terms between worms treated with control (empty vector) and ash-2

1219 RNAi. GO terms were analyzed using WormEnrichR ${ }^{29,123}$. Left column: GO terms upregulated

1220 in middle aged individuals (post-reproductive, adult day 6/ whole worms) ${ }^{5}$. Right column: GO

1221 terms upregulated in young individuals (adult day $1 /$ intestine) ${ }^{6}$. GO terms were considered

1222 significant if they have a combined score of $\log P$-value (Fisher's exact test) multiplied with 1223 rank-based enrichment $\mathrm{z}$-score larger than $5\left(\operatorname{Ref}^{122-124}\right)$. See Extended Data Table 2 for all GO

1224 terms and classifications.

1225 c-d, Peroxisome number increases in MUFA-enriched worms. Intestinal peroxisomes measured 1226 by fluorescence in the ges-1p::GFP-SKL transgenic line, which expresses a peroxisome-

1227 localized GFP driven by the intestinal ges-1 promoter. c, Zoomed-in fluorescent images of the 1228 last intestinal cell in worms treated with control (empty vector) RNAi or ash-2 or fat-2 RNAi 1229 (MUFA-enriched). Scale bar $=5 \mu \mathrm{m}$. Intensity of peroxisome-localized GFP quantified in 1230 Extended Data Fig 3a.

1231 d, Quantification of peroxisome number in worms treated with control (empty vector) RNAi or 1232 ash-2 or fat-2 RNAi (MUFA-enriched). $n \geq 35$ worms for each condition. Representative of three 1233 independent experiments (see Source Data Figure 3 for all experiment data and statistics). Data 1234 are mean \pm s.d. Each dot represents the peroxisome number in a $26 \times 26 \mu \mathrm{m}^{2}$ area in the intestine 1235 of an individual worm. $P$-values: two-tailed Mann-Whitney test.

1236 e, prx-5 is necessary for longevity upon MUFA enrichment by ash-2 depletion. Kaplan Meier 1237 survival curve of worms treated with control (empty vector), ash-2, prx-5, or ash-2/prx-5 RNAi. $1238 \mathrm{n} \geq 94$ for each condition. Representative of two independent experiments (see Extended Data 1239 Table 1 for all lifespan experiments and statistics). Percentages of median lifespan extension and $1240 P$-values are indicated on the right. $P$-values: log-rank Mantel-Cox test. ash-2 and prx-5 RNAi 1241 significantly interact with each other using the Cox proportional hazard test $(P$-value $=4.02 \mathrm{e}-06)$.

1242 f, Lipid droplet and peroxisome accumulation/degradation follow similar dynamics with age. 1243 Intestinal peroxisomes and lipid droplets measured by fluorescence in the $d h s-3 p:: d h s-3:: G F P$, $1244 v h a-6 p:: m R F P-S K L$ transgenic line in MUFA-enriched worms. This transgenic line expresses 
both the lipid droplet marker DHS-3 fused to GFP driven by the $d h s-3$ promoter (intestine) and a peroxisome localized-mRFP driven by the vha-6 promoter (intestine). Quantification of organelle numbers in worms treated with control (empty vector) or ash-2 RNAi. $n \geq 23$ worms for each condition. Representative of two independent experiments (see Source Data Figure 3 for all experiment data and statistics). Each dot represents the mean organelle number in a 26 × 26 $\mu \mathrm{m}^{2}$ area in the intestine of all worms imaged for this condition. Left y-axis: lipid droplet number, right y-axis: peroxisome number.

g, Design of targeted screen to test for effect of specific genes on lipid droplet and peroxisome number.

h, Lipid droplet and peroxisome numbers correlate in the screen. Intestinal peroxisomes and lipid droplets measured by fluorescence in the $d h s-3 p:: d h s-3:: G F P$, vha-6p::mRFP-SKL transgenic line. Quantification of organelle numbers in worms treated with 50 different RNAis (see Source Data Figure 3 for all experiment data and statistics). $n \geq 15$ worms for each condition. Each dot represents the mean organelle number in a $26 \times 26 \mu \mathrm{m}^{2}$ area in the intestine of all worms imaged for this condition normalized to the control mean. Black: Control (empty vector) RNAi. Red: RNAi conditions that increase longevity. Blue: RNAi conditions that decrease longevity upon MUFAs. White: RNAi conditions that have no effects on longevity or were not tested. Correlation: Pearson $\mathrm{R}^{2}$ test.
$\mathbf{i}, \mathbf{j}$ Genes that influence lipid droplet number. $\mathbf{i}$, Intestinal lipid droplets measured by fluorescence in the dhs-3p::dhs-3::GFP, vha-6p::mRFP-SKL transgenic line. Quantification of lipid droplet number in worms treated with 50 different RNAis (see Source Data Figure 3 for all experiment data and statistics). $n \geq 15$ worms for each condition. Data are mean \pm s.d. Each dot represents the organelle number in a $26 \times 26 \mu \mathrm{m}^{2}$ area in the intestine of an individual worm normalized to control worms. Orange: significant increase in lipid droplet number, light blue: significant decrease in lipid droplet number. Conditions are colored if the adjusted $P$-value $<$ 0.05 using the unpaired Wilcoxon test with Benjamini-Hochberg test for multiple hypothesis correction. (*) dhs-3 RNAi abolishes the GFP signal of the lipid droplet DHS-3::GFP reporter. $\mathbf{j}$, Genes that regulate of lipid droplet numbers found in i. Orange: significant increase in lipid droplet number, light blue: significant decrease in lipid droplet number.

$\mathbf{k}, \mathbf{l}$ Genes that influence peroxisome number. $\mathbf{k}$, Intestinal peroxisome measured by fluorescence in the $d h s-3 p:: d h s-3:: G F P$, vha-6p::mRFP-SKL transgenic line. Quantification of peroxisome number in worms treated with 50 different RNAis (see Source Data Figure 3 for all experiment 
1277 data and statistics). $n \geq 19$ worms for each condition. Data are mean \pm s.d. Each dot represents

1278 the organelle number in a $26 \times 26 \mu \mathrm{m}^{2}$ area in the intestine of an individual worm normalized to

1279 control worms. Orange: significant increase peroxisome number, light blue: significant decrease

1280 in peroxisome number. Conditions are colored if the adjusted $P$-value $<0.05$ using the unpaired

1281 Wilcoxon test with Benjamini-Hochberg test for multiple hypothesis correction. 1, Genes that

1282 regulate of peroxisome numbers found in k. Orange: significant increase in peroxisome number,

1283 light blue: significant decrease in peroxisome number.

$1284 \mathbf{m}, a d s-1$, a gene involved in ether lipid synthesis, is necessary for longevity upon MUFA

1285 enrichment by ash-2 depletion. Kaplan Meier survival curve of worms treated with control

1286 (empty vector), ash-2, ads-1, or $a s h-2 / a d s-1$ RNAi. $\mathrm{n} \geq 81$ for each condition. Representative of

1287 two independent experiments (see Extended Data Table 1 for all lifespan experiments and

1288 statistics). Percentages of median lifespan extension and $P$-values are indicated on the right. $P$ -

1289 values: log-rank Mantel-Cox test. ash-2 and ads-1 RNAi significantly interact with each other

1290 using the Cox proportional hazard test $(P$-value $=1.28 \mathrm{e}-07)$.

1291 n, $a d s-1$, a gene involved in ether lipid synthesis, is necessary for longevity upon lipid droplet

1292 enrichment by hosl-1 depletion. Kaplan Meier survival curve of worms treated with control

1293 (empty vector), hosl-1, ads-1, or hosl-1/ads-1 RNAi. $\mathrm{n} \geq 94$ for each condition. Representative of

1294 two independent experiments (see Extended Data Table 1 for all lifespan experiments and

1295 statistics). Percentages of median lifespan extension and $P$-values are indicated on the right. $P$ -

1296 values: log-rank Mantel-Cox test. hosl-1 and ads-1 RNAi significantly interact with each other

1297 using the Cox proportional hazard test $(P$-value $=1.23 \mathrm{e}-06)$. Control lifespan curves are also

1298 shown in Fig. 2h.

1299 All experiment data and statistics are in Source Data Figure 3. All lifespan data are in Extended

1300 Data Table 1.

1301 
a Knock-down of genes until middle-age



Untargeted Lipidomics (LC/MS) b Fatty acid abundance among all lipids



C

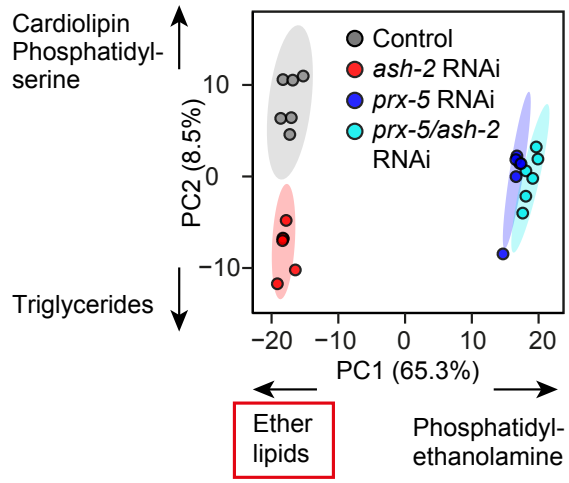

d

Phospholipid membrane with Ether lipid

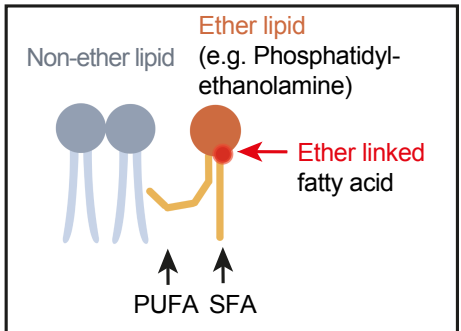

Ether linked fatty acids are also detected in triglycerides e

Fatty acid abundance among all Ether lipids

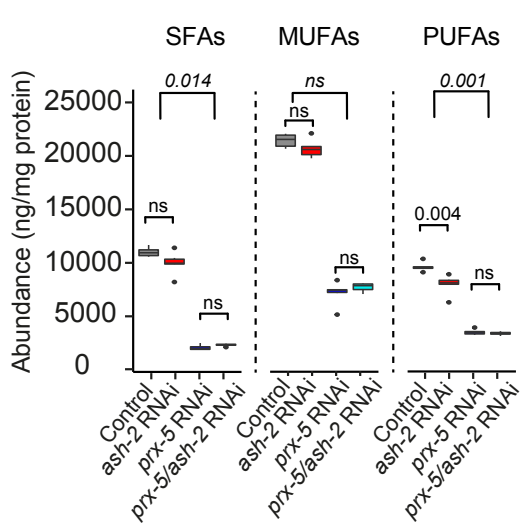

f

MUFA to PUFA ratio among Ether lipids

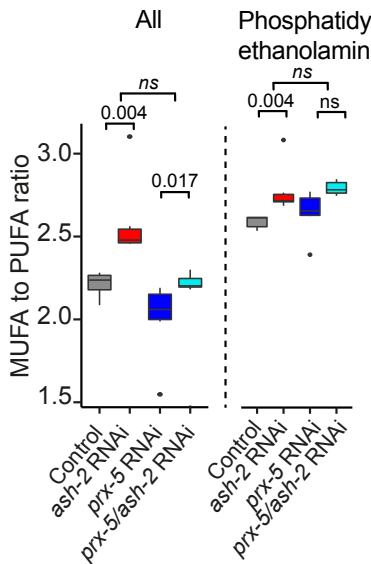

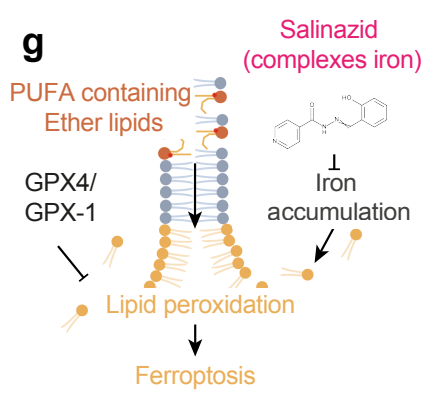

\section{h}

i
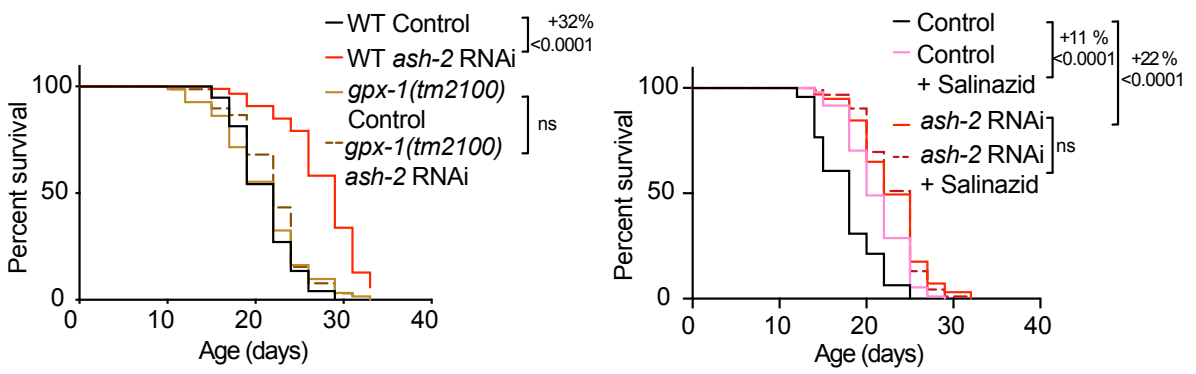

j

k

Ferroptosis protection (Salinazid) and lipid droplet disruption (Ipin-1 RNAi)

Ferroptosis protection (Salinazid) and Ether lipid disruption (ads-1 RNAi)
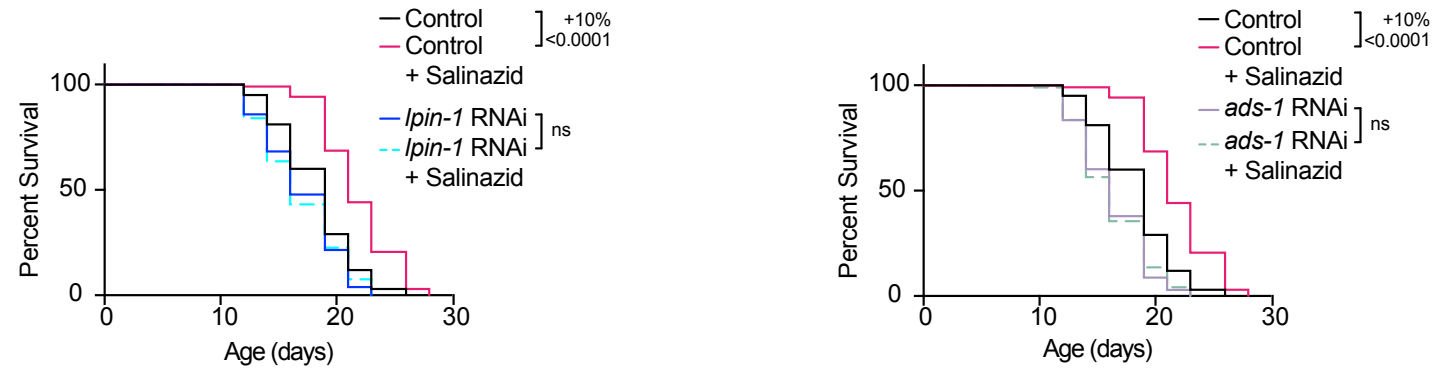
1304

1305

1306

1307

1308

1309

1310

1311

1312

1313

1314

1315

1316

1317

1318

1319

1320

1321

1322

1323

1324

1325

1326

1327

1328

1329

1330

1331

1332

1333

1334

1335

Figure 4. Ether lipid changes upon MUFA supplementation and role of ferroptosis in MUFA-mediated longevity

a, Untargeted lipidomic analysis on whole worms using liquid chromatography/mass spectrometry (LC/MS). Middle-aged worms (adult day 6) treated with control (empty vector), ash-2, prx-5, or ash-2/prx-5 RNAi were lyzed, and whole-worm lysates were subjected to LC/MS. Six biological replicates with $\sim 500$ individual worms per condition.

b, Fatty acid abundance of saturated fatty acids (SFAs), monounsaturated fatty acids (MUFAs) and polyunsaturated fatty acids (PUFAs) among all lipids in middle-aged worms treated with control (empty vector), ash-2, prx-5, or ash-2/prx-5 RNAi. Box and whisker plot, with median (central line), $25^{\text {th }}$ and $75^{\text {th }}$ percentile (outer lines), and minimum and maximum within 1.5 times the interquartile range (whiskers). Values beyond these are considered outliers and plotted individually. Complete list of fatty acid categories and statistics is in (Source Data Figure 4). $P$-values: Unpaired two-samples Wilcoxon test with Benjamini-Hochberg test for multiple hypothesis correction. Italic $P$-values: two-way ANOVA.

c, Principal component analysis (PCA) on the lipidome separates MUFA-enriched conditions (ash-2 RNAi) from control conditions and from conditions in which peroxisomes are depleted (prx-5 and prx-5/ash-2 RNAi). Each dot represents a biological replicate. Six biological replicates of $\sim 500$ individual worms per condition. Complete list of all lipids driving the PCA separation available is in (Extended Data Table 3).

d, Ether lipids are mostly located in membranes. Ether lipids contain one fatty acid that is attached via an ether-bond to the head group (red circle). Ether lipids are present in several lipid classes, but are enriched in membrane lipids (e.g. phosphatidylethanolamine, PE). Complete list of detected ether lipids and statistics is in (Source Data Extended Data Figure 4).

e, Fatty acid abundance of saturated fatty acids (SFAs), monounsaturated fatty acids (MUFAs) and polyunsaturated fatty acids (PUFAs) among ether lipids in middle-aged worms treated as in a. Box and whisker plot, with median (central line), $25^{\text {th }}$ and $75^{\text {th }}$ percentile (outer lines), and minimum and maximum within 1.5 times the interquartile range (whiskers). Values beyond these are considered outliers and plotted individually. Complete list of fatty acid categories and statistics is in (Source Data Figure 4). $P$-values: Unpaired two-samples Wilcoxon test with Benjamini-Hochberg test for multiple hypothesis correction. Italic $P$-values: two-way ANOVA. f, MUFA to PUFA ratio among all ether lipids (left panel) and among ether lipids in phosphatidylethanolamine (PE, right panel) in worms treated as in a. Data and statistics as in b. 
1336

1337

1338

1339

1340

1341

1342

1343

1344

1345

1346

1347

1348

1349

1350

1351

1352

1353

1354

1355

1356

1357

1358

1359

1360

1361

1362

1363

1364

1365

1366

The PE panel is also in Extended Data Fig. 4e. Complete list of the MUFA to PUFA ratios and statistics can be found in (Source Data Figure 4).

g, Strategies to inhibit ferroptosis. GPX4/GPX-1 protects cells against membrane peroxidation by degrading lipid peroxides. The chemical Salinazid complexes iron, thereby blocking toxic iron accumulation and ferroptosis.

h, gpx-1 is necessary for longevity upon MUFA enrichment by ash-2 depletion. Kaplan Meier survival curve of gpx-1(tm21000) or wildtype worms treated with control (empty vector) or ash2 RNAi. $\mathrm{n} \geq 82$ for each condition. Representative of two independent experiments (see Extended Data Table 1 for all lifespan experiments and statistics). Percentages of median lifespan extension and $P$-values are indicated on the right. $P$-values: log-rank Mantel-Cox test. Gpx-1(tm21000) and ash-2 RNAi significantly interact with each other using the Cox proportional hazard test $(P$-value $=3.4 \mathrm{e}-09)$.

i, Ferroptosis protection does not further increase MUFA-mediated longevity. Kaplan Meier survival curve of worms treated with control (empty vector) or ash-2 RNAi upon Salinazid. $\mathrm{n} \geq$ 110 for each condition. Percentages of median lifespan extension and $P$-values are indicated on the right. $P$-values: log-rank Mantel-Cox test. $a s h-2$ RNAi and Salinazid significantly interact with each other using the Cox proportional hazard test $(P$-value $=2.10 \mathrm{e}-06)$. Representative of one independent experiment (see Extended Data Table 1 for all lifespan experiments and statistics). Controls are also plotted in Extended Data Fig. 4f.

j, Lipid droplets are necessary for longevity upon ferroptosis inhibition by Salinazid. Kaplan Meier survival curve of worms treated with control (empty vector) or lpin-1 RNAi upon Salinazid. $\mathrm{n} \geq 105$ for each condition. Percentages of median lifespan extension and $P$-values are indicated on the right. $P$-values: log-rank Mantel-Cox test. Lpin-1 RNAi and Salinazid significantly interact with each other using the Cox proportional hazard test $(P$-value $=1.79 \mathrm{e}-05)$. Representative of two independent experiments (see Extended Data Table 1 for all lifespan experiments and statistics). Controls are also plotted in $\mathrm{k}$.

k, Ether lipids are necessary for longevity upon ferroptosis inhibition by Salinazid. Kaplan Meier survival curve of worms treated with control (empty vector) or ads-1 RNAi upon Salinazid. $\mathrm{n} \geq$ 105 for each condition. Percentages of median lifespan extension and $P$-values are indicated on the right. $P$-values: log-rank Mantel-Cox test. $a d s-1$ RNAi and Salinazid significantly interact with each other using the Cox proportional hazard test $(P$-value $=4.41 \mathrm{e}-05)$. Representative of 
1367 two independent experiments (see Extended Data Table 1 for all lifespan experiments and

1368 statistics). Controls are also plotted in $\mathrm{j}$.

1369 All experiment data and statistics are in Source Data Figure 4. All lifespan data and statistics are 1370 in Extended Data Table 1.

1371 


\section{Supplementary Files}

This is a list of supplementary files associated with this preprint. Click to download.

- KP20210622ExtendedDataTable1Lifespandata.xlsx

- KP20210618ExtendedDataTable2GOterms.xIsx

- KP20210618ExtendedDataTable3PCAloadings.xlsx

- KP20210621ExtendedDataTable4Wormstrains.xIsx

- KP20210621ExtendedDataTable5qPCRprimer.xlsx

- KP20210621ExtendedDataTable6LipidomicData.xlsx

- KP20210720ExtendedDataTable7Fattyacidlength.xIsx

- PapsdorfetalExtendedData.pdf 